\title{
Principal Chiral Model on Superspheres
}

\author{
Vladimir Mitev ${ }^{1}$, Thomas Quella ${ }^{2}$ and Volker Schomerus ${ }^{1}$ \\ ${ }^{1}$ DESY Hamburg, Theory Group, \\ Notkestrasse 85, D-22607 Hamburg, Germany \\ 2 Institute for Theoretical Physics, University of Amsterdam, \\ Valckenierstraat 65, 1018 XE Amsterdam, The Netherlands
}

\begin{abstract}
We investigate the spectrum of the principal chiral model (PCM) on odd-dimensional superspheres as a function of the curvature radius $R$. For volume-filling branes on $S^{3 \mid 2}$, we compute the exact boundary spectrum as a function of $R$. The extension to higher dimensional superspheres is discussed, but not carried out in detail. Our results provide very convincing evidence in favor of the strong-weak coupling duality between supersphere PCMs and $\operatorname{OSP}(2 S+2 \mid 2 S)$ Gross-Neveu models that was recently conjectured by Candu and Saleur.
\end{abstract}

e-mail: Vladimir.Mitev@desy.de,T.Quella@uva.nl, Volker.Schomerus@desy.de 


\section{Contents}

1 Introduction 1

2 Spectrum of the supersphere PCM at large volume 6

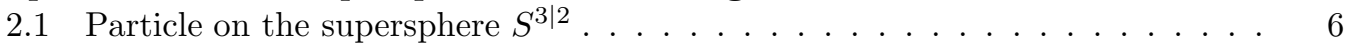

2.2 The complete boundary spectrum . . . . . . . . . . . . . . . . . 9

2.3 Casimir decomposition of the boundary spectrum . . . . . . . . . 13

3 The $\operatorname{OSP}(4 \mid 2)$ GN model and the supersphere $\mathrm{S}^{3 \mid 2} \quad 17$

3.1 Free field construction of the bulk theory . . . . . . . . . . . . . 17

3.2 Boundary conditions and their spectra . . . . . . . . . . . . . 21

3.3 Casimir decomposition in the free GN model . . . . . . . . . . . . . 23

3.4 Deformation from free $\mathrm{GN}$ model to free $\mathrm{PCM} \ldots \ldots . \ldots . . . . .25$

4 Generalization for higher-dimensional superspheres

4.1 Partition functions for superspheres at $R=1, \infty \ldots \ldots \ldots \ldots$

4.2 Test of the duality . . . . . . . . . . . . . . . 29

5 Conclusions, open questions and outlook 31

A Some aspects of the representation theory of $\operatorname{OSP}(4 \mid 2)$

B Some useful identities 36

B.1 Identities used in the Casimir decomposition . . . . . . . . . . 36

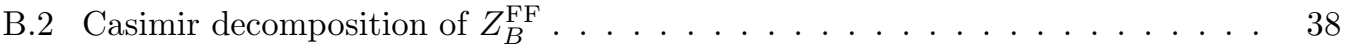

C Recombination of the bosonic characters 39

D A free field construction for $\widehat{\operatorname{osp}}(\mathrm{M} \mid 2 \mathrm{~N})_{1}$

\section{Introduction}

Principal chiral models (PCMs) on symmetric spaces have been studied extensively because of their numerous applications in many different branches of physics. While PCMs on symmetric spaces are well-known to possess an infinite number of classically conserved quantities (see [1, 2, 3, 4, 5, 6, for early work and e.g. [7, 8, for more recent developments and references), quantum effects spoil integrability in many cases [9, 10. And even in those examples for which this does not happen, finding explicit formulas for partition functions and correlators is a difficult problem that has only been solved for a small set of models. More recently, PCMs on (generalized) symmetric superspaces have received considerable attention. This is explained in part through the role they play for the description of strings for Anti-de Sitter (AdS) backgrounds in various dimensions, including $A d S_{5} \times S^{5}$ and $A d S_{4} \times \mathbb{C P}^{3}$ [11, 12, 13, 14]. PCMs on symmetric superspaces possess a number of remarkable properties. In particular, there exist several families of quantum conformal models [15, 16, 17, 18, 19]. Yet, finding explicit solutions is still rather difficult and will certainly require developing new techniques, see e.g. 20, 21]. Some remarkable recent advances, most importantly the results of [22] and 
23, 24], seem to bring at least some partial solutions within reach. One of our aims here is to initiate and explore new solution strategies that incorporate target space supersymmetry as an essential feature.

In this work we focus on a particular family of symmetric target superspaces, namely on the odd dimensional superspheres $S^{2 S+1 \mid 2 S}$ with $2 S$ fermionic coordinates. The supersphere $S^{2 S+1 \mid 2 S}$ admits at least three different descriptions that will be somewhat useful for us below. We can think of $S^{2 S+1 \mid 2 S}$ as a supermanifold in $\mathbb{R}^{2 S+2 \mid 2 S}$ defined by the equation

$$
\sum_{i=1}^{2 S+2} x_{i}^{2}+2 R^{2} \sum_{a=1}^{S} \eta_{2 a-1} \eta_{2 a}=R^{2}
$$

Here, $x_{i}, i=1, \ldots, 2 S+2$, and $\eta_{j}, j=1, \ldots, 2 S$, are the bosonic and fermionic coordinates of $\mathbb{R}^{2 S+2 \mid 2 S}$, respectively. The real parameter $R$ has been introduced to denote the radius of the supersphere. Note that in our conventions, the bosonic coordinates scale with the length while the fermionic coordinates are chosen to be dimensionless. From our description of the supersphere through equation (1.1) it is evident that $S^{2 S+1 \mid 2 S}$ comes equipped with an $\operatorname{osp}(2 S+2 \mid 2 S)$ action. In fact, the Lie superalgebra $\operatorname{osp}(2 S+2 \mid 2 S)$ acts on the embedding space $\mathbb{R}^{2 S+2 \mid 2 S}$ through its fundamental representation. By the very definition of $\operatorname{OSP}(2 S+$ $2 \mid 2 S)$ this action respects the constraint (1.1). Hence, we arrive at a second description of $S^{2 S+1 \mid 2 S}$ as a symmetric space

$$
S^{2 S+1 \mid 2 S}=\operatorname{OSP}(2 S+2 \mid 2 S) / \mathrm{OSP}(2 S+1 \mid 2 S) .
$$

Note that the stabilizer of any point on the supersphere is isomorphic to the subsupergroup $\operatorname{OSP}(2 S+1 \mid 2 S) \subset \operatorname{OSP}(2 S+2 \mid 2 S)$. Finally, we can also solve the constraint (1.1) explicitly by parametrizing the supersphere $S^{2 S+1 \mid 2 S}$ through $2 S+1$ angular coordinates $\varphi_{j}$ and $2 S$ fermionic variables $\eta_{j}$. In the case of the 3 -sphere $S^{3 \mid 2}$, for example, the line element takes the following form

$$
d s^{2}=2 R^{2}\left(1-\eta_{1} \eta_{2}\right) d \eta_{1} d \eta_{2}+R^{2}\left(1-2 \eta_{1} \eta_{2}\right) d \Omega_{3}
$$

where

$$
d \Omega_{3}=d \varphi_{1}^{2}+\cos ^{2} \varphi_{1} d \varphi_{2}^{2}+\sin ^{2} \varphi_{1} d \varphi_{3}^{2}
$$

is the usual line element of the 3-dimensional unit sphere. All three descriptions of the supersphere $S^{2 S+1 \mid 2 S}$ will be used frequently throughout the rest of this work.

Next we turn to the principal chiral model on the supersphere. Once more, there are different ways to introduce this theory. The most basic one is to think of it as a linear sigma model for the fields $x_{i}$ and $\eta_{j}$ with a non-linear constraint (1.1) on the field configurations. Another possibility is to consider it as a non-linear sigma model. In the case of the 3dimensional supersphere the latter takes the form

$$
\begin{aligned}
\mathcal{S}^{\mathrm{PCM}}=\frac{R^{2}}{2 \pi} \int d^{2} z & \left(2\left(1-\eta_{1} \eta_{2}\right)\left(\partial \eta_{1} \bar{\partial} \eta_{2}-\partial \eta_{2} \bar{\partial} \eta_{1}\right)\right. \\
+ & \left.\left(1-2 \eta_{1} \eta_{2}\right)\left(\partial \varphi_{1} \bar{\partial} \varphi_{1}+\cos ^{2} \varphi_{1} \partial \varphi_{2} \bar{\partial} \varphi_{2}+\sin ^{2} \varphi_{1} \partial \varphi_{3} \bar{\partial} \varphi_{3}\right)\right)
\end{aligned}
$$


for the fields $\eta_{j}, \varphi_{i}$. The coupling constant in front of the action is determined by the radius $R$ of $S^{3 \mid 2}$. For the PCM on the purely bosonic 3-sphere the coupling $R$ runs and in order for the flow to end in a non-trivial fixed-point one must add a WZ term [25]. But the presence of the two fermionic directions changes the situation drastically. As shown in [18, the $\beta$ function of the PCM on $S^{2 S+1 \mid 2 S}$ is the same as for a bosonic PCM on a sphere $S^{d}$ whose dimension $d=2 S+1-2 S=1$ is given by the difference between the number of bosonic and fermionic coordinates. Consequently, the $\beta$-function vanishes for the PCM on $S^{2 S+1 \mid 2 S}$, i.e. the model (1.4) defines a family of conformal field theories at central charge $c=1$ with continuously varying exponents.

Of course, unlike the PCM on $S^{1}=\mathrm{U}(1)$, the theory defined by the action (1.4) is not free. For large radius $R$, the model is weakly coupled and its properties may by studied perturbatively. But as we pass to a more strongly curved background, computing quantities as a function of the radius $R$ may seem like a very daunting task. This is even more so because there is very little symmetry to work with. As a conformal field theory, the PCM on the supersphere possesses the usual chiral Virasoro symmetries. But for a model with multiple bosonic coordinates the two sets of chiral Virasoro generators are not sufficient to make the theory rational. In addition, there is a single set of global osp(4|2) generators. Their Noether currents, however, fail to be chiral, at least for generic points in the moduli space. Without the protection of current algebra symmetries, the usual algebraic tools of conformal field theory cannot be applied to supersphere PCMs and so we have to proceed along a rather different route.

Many years of experience with sigma models show that they often possess interesting dual descriptions. The simplest such duality is that between the free compactified boson and the massless Thirring model. Let us recall that the latter involves two real fermions $\psi_{1}$ and $\psi_{2}$ and the following action

$$
\mathcal{S}_{m=0}^{\mathrm{Th}}=\frac{1}{2 \pi} \int d^{2} z \sum_{i=1}^{2}\left[\psi_{i} \bar{\partial} \psi_{i}+\bar{\psi}_{i} \partial \bar{\psi}_{i}+g^{2}\left(\psi_{1} \bar{\psi}_{2}-\psi_{2} \bar{\psi}_{1}\right)^{2}\right]
$$

where the compactification radius $R$ is related to the coupling $g$ through $R^{2}=1+g^{2}$. Similarly, one may hope to uncover a dual description of the PCM on the supersphere $S^{2 S+1 \mid 2 S}$ that becomes weakly coupled for some finite value of the radius $R$, deep in the strongly curved regime. Such a dual description was indeed proposed recently. According to an intriguing conjecture by Candu and Saleur [24, there indeed exists one special radius $R=R_{0}$ at which the PCM on $S^{2 S+1 \mid 2 S}$ can be described as a non-interacting Gross-Neveu model involving $2 S+2$ real fermions $\psi_{i}$ along with $S$ bosonic $\beta \gamma$ systems $\gamma_{a}$ and $\beta_{a}$,

$$
\mathcal{S}_{g=0}^{\mathrm{GN}}=\frac{1}{2 \pi} \int d^{2} z\left[\sum_{i}\left(\psi_{i} \bar{\partial} \psi_{i}+\bar{\psi}_{i} \partial \bar{\psi}_{i}\right)+\sum_{a}\left(\beta_{a} \bar{\partial} \gamma_{a}+\bar{\beta}_{a} \partial \bar{\gamma}_{a}\right)\right] .
$$

All the fields appearing in this theory possess conformal weight $h_{i}=h_{a}=1 / 2$ so that the central charge is $c=S+1-S=1$. At this point in the moduli space, the theory possesses two 
commuting sets of chiral osp $(4 \mid 2)$ currents $J^{\mu}=J^{\mu}(z)$ and $\bar{J}^{\mu}=\bar{J}^{\mu}(\bar{z})$. Explicit formulas will be spelled out in section 3 below. The affine symmetry is broken down to a global $\operatorname{osp}(4 \mid 2)$ symmetry by the following osp(4|2) invariant marginal deformation

$$
\mathcal{S}^{\text {int }}=\frac{g^{2}}{2 \pi} \int d^{2} z J_{\mu}(z) \Omega\left(\bar{J}^{\mu}(\bar{z})\right)=\frac{g^{2}}{2 \pi} \int d^{2} z\left[\sum_{i} \varpi_{i} \psi_{i} \bar{\psi}_{i}+\sum_{a}\left(\gamma_{a} \bar{\beta}_{a}-\beta_{a} \bar{\gamma}_{a}\right)\right]^{2} .
$$

Here, $\Omega$ is a particular automorphism of the $\operatorname{osp}(2 \mathrm{~S}+2 \mid 2 \mathrm{~S})$ current algebra which leaves a subalgebra osp $(2 \mathrm{~S}+1 \mid 2 \mathrm{~S})$ invariant. It will be spelled out explicitly below. The numbers $\varpi_{i}$ are given by $\varpi_{1}=-1$ and $\varpi_{i}=1$ for $i \neq 1$. The theory $\mathcal{S}^{\mathrm{GN}}=\mathcal{S}_{g=0}^{\mathrm{GN}}+\mathcal{S}^{\mathrm{int}}$ is claimed to be equivalent to the supersphere PCM with the two coupling constants $R$ and $g$ related by $R^{2}=1+g^{2} 1$ The equivalence is a strong-weak coupling duality since $\mathcal{S}^{\mathrm{GN}}$ becomes weakly coupled for $R \sim R_{0}=1$. Note that this duality is a direct generalization of the relation between the compactified free field and the massless Thirring model. There appears one real fermion for each bosonic coordinate of the embedding space $\mathbb{R}^{2 S+2 \mid 2 S}$. Each pair of additional fermionic directions gives rise to a $\beta \gamma$ system. Note, however, that the duality between supersphere PCMs and Gross-Neveu models is one between interacting conformal field theories. In that sense, it is much less trivial then its purely bosonic counterpart.

The main aim of this note is to provide very compelling evidence for the duality between the theory (1.5[1.6) and the supersphere PCMs, extending previous numerical and algebraic arguments given in 23, 24. To this end we shall employ some recent results of 22] that are designed to compute exact spectra in models with a special class of target space supersymmetries, including the two series $\operatorname{psl}(\mathrm{N} \mid \mathrm{N})$ and $\operatorname{osp}(2 S+2 \mid 2 S)$. The Lie superalgebra $\operatorname{osp}(2 S+2 \mid 2 S)$ possesses a vanishing quadratic Casimir $C_{\text {ad }} \sim f_{\mu \nu \rho} f^{\mu \nu \rho}$ in the adjoint representation. Since $C_{\text {ad }}$ may be considered as a rough measure for the 'amount of non-abelianess' of a Lie superalgebra, one may suspect that field theories with osp $(2 S+2 \mid 2 S)$ symmetry are somewhat intermediate between free field theories and the most general interacting models. Indeed, as was shown in [16, 22, the perturbation series for conformal weights has features that are very reminiscent of those in abelian models (torus compactifications). In this note we shall construct the exact partition function of the theory (1.5]1.6) with a particular choice of boundary conditions, but for all values(!) of the coupling $g$. We shall prove that it interpolates correctly between $g=0$ and the spectrum of the supersphere PCM at $R=\infty$.

The main results of 22] are rather easy to state. Before we do so, let us briefly review the behavior of conformal weights for a compactified free bosonic field $\varphi \sim \varphi+2 \pi R$. Suppose we are given a field $\Psi$ of conformal weight $h_{0}(\Psi)$ at some radius $R_{0}$. In order to find the conformal weight of the same field $\Psi$ at a different radius $R$, it suffices to know its $\mathrm{U}(1)$ charge $g(\Psi)$ (momentum/winding). The conformal weight is then given by

$$
h(\Psi)=h_{0}(\Psi)+f(R) g^{2}(\Psi)
$$

\footnotetext{
${ }^{1}$ Let us note that the signs $\varpi_{i}$ in the iteraction term are directly linked to the automorphism $\Omega$. These signs were missing in the original formulation of the conjecture by Candu and Saleur 24]. They are irrelevant for $S=0$ but play a certain role when $S \geq 1$.
} 
where $f(R)$ is some universal function of the radius that is the same for all fields $\Psi . f(R)$ may depend, however, on whether $\Psi$ is a bulk or boundary field and on the precise boundary condition that is imposed. For bulk fields, there exist independent left and right U(1) charges and the behavior of the weights is a bit more complicated. We shall briefly comment on this issue in the conclusions. Returning to our supersphere conformal field theories, we pick any field $\Psi$ of weight $h_{0}(\Psi)$ in the free field theory (1.5). Let us suppose that $\Psi$ is part of some $\operatorname{osp}(2 S+2 \mid 2 S)$ multiplet $\Lambda$. According to the arguments explained in [22] (see also [23] for numerical checks), its dimension at radius $R$ is then given by

$$
h(\Psi)=h_{0}(\Psi)+f(R) C_{2}(\Lambda) .
$$

Here, $C_{2}(\Lambda)$ is the value of the quadratic Casimir element in the representation $\Lambda$ of the Lie superalgebra $\operatorname{osp}(2 S+2 \mid 2 S)$. Once again, the function $f(R)$ is universal, i.e. it does not depend on the field $\Psi$. Hence, the shift of the conformal weight is entirely determined by the way $\Psi$ transforms under the action of the Lie superalgebra $\operatorname{osp}(2 S+2 \mid 2 S)$. Equation (1.8) is the direct generalization of eq. (1.7) with the square of the $\mathrm{U}(1)$ charge replaced by the quadratic Casimir. The behavior (1.8) has been also been predicted through the study of lattice algebras in [24]. It was furthermore checked using perturbative calculations at $R=\infty$ and with numerical simulations. We shall refer to the behavior (1.8) as a quasi-abelian deformation of conformal weights. It is typical for models with $\operatorname{osp}(2 S+2 \mid 2 S) \operatorname{or} \operatorname{psl}(\mathrm{N} \mid \mathrm{N})$ symmetry, though often restricted to particular (boundary) fields of the theories (see [22] and final section for more details). Let us mention that fields transforming in representations with vanishing Casimir $C_{2}(\Lambda)$ are protected, i.e. their conformal weights are independent of $R$. Multiplets of this type always satisfy some shortening conditions. Our formula (1.8), however, applies to all fields in the theory, irrespectively of whether they are long or short. It allows to compute their conformal weight for all values of the radius $R$.

Let us study a few concrete examples of the quasi-abelian deformation of conformal weights. In the large volume limit, the PCM possesses an infinite number of fields with conformal weight $h=0$. These simply correspond to functions on the supersphere. The simplest function is the constant. Since it transforms in the trivial representation of $\operatorname{osp}(2 S+2 \mid 2 S)$, its conformal weight remains undeformed at $h=0$. It corresponds to the unique vacuum state of the free Gross-Neveu model (1.5). Next, the PCM contains the fundamental multiplet $x_{i}, \eta_{j}$. The quadratic Casimir of this multiplet $\Lambda=\Lambda_{f}$ is $C_{2}\left(\Lambda_{f}\right)=1$, i.e. its value is independent of $S$. As we move from the free sigma model at $R=\infty$ towards the free Gross-Neveu model (1.5), the fields $x_{i}, \eta_{j}$ acquire a non-vanishing anomalous dimension which becomes $h=h_{0}+f\left(R_{0}\right) C_{2}\left(\Lambda_{f}\right)=1 / 2$ when we reach the radius $R=1$ corresponding to $g=0$. Hence, the fundamental multiplet of the PCM turns into the multiplet $\psi_{i}, \gamma_{a}, \beta_{a}$. Higher functions possess larger Casimir and hence they are mapped to states of weight $h>1 / 2$ at $g=0$. Beyond the space of ground states in the PCM, there are fields involving any number of world-sheet derivatives. These have positive integer weight at $R=\infty$. As we shall see below, such states can transform in $\operatorname{osp}(2 S+2 \mid 2 S)$ representations $\Lambda$ with both positive and 
negative values $C_{2}(\Lambda)$ of the quadratic Casimir. Consequently, some of these multiplets are moved up while others are moved down to lower weights. Our claim is that weights are rearranged in precisely the right way to reproduce the spectrum of the $g=0$ Gross-Neveu model.

The plan of this work is as follows. In the next section we shall study the PCM (1.4) for the 3-dimensional supersphere $S^{3 \mid 2}$ and determine its exact spectrum at $R=\infty$. For simplicity, we shall also restrict to the partition function on a strip with Neumann boundary conditions imposed along both boundaries. After a detailed discussion of the low lying states, we present a closed formula for the full partition function (2.16). The latter is then decomposed explicitly into the contributions coming from states which transform in the same representation $\Lambda$ under the global $\operatorname{osp}(4 \mid 2)$. Section 3 is devoted to the theory (1.5) and its deformation by the term (1.6). In particular, we study the bulk and boundary spectrum of the free field theory. One of the resulting boundary partition functions is then expanded explicitly in terms of osp $(4 \mid 2)$ characters. This allows us to compare with the spectrum of the PCM at radii $R<\infty$, using some of the tools developed in 22. We shall find that the results agree exactly with the partition function found in section 2 ! In the fourth section, we comment on the generalization to higher dimensional superspheres. Finally, the conclusions contain a few general thoughts on possible implications for string theory in Anti-deSitter spaces. We shall also briefly discuss the computation of bulk spectra for odd dimensional superspheres.

\section{Spectrum of the supersphere PCM at large volume}

In this section we shall focus on the PCM for the supersphere $S^{3 \mid 2}$ with large radius $R$. At the point $R=\infty$ we can compute partition functions for periodic boundary conditions and on a strip. The two main ingredients are the exact minisuperspace spectrum on $S^{3 \mid 2}$ (see subsection 2.1) and a good control of the combinatorics that determine the field theoretic spectrum at $R=\infty$. The latter will be explained in subsection 2.2. The spectrum is finally decomposed into finite dimensional representations of the global symmetry algebra osp $(4 \mid 2)$ in the third subsection.

\subsection{Particle on the supersphere $S^{3 \mid 2}$}

The Laplacian on the supersphere $S^{3 \mid 2}$ was analyzed in full detail by Candu and Saleur [24]. We shall state their results first and then provide a new derivation that is particularly well suited for the discussion in the following subsections.

As a warm-up, let us briefly recall the spectrum of the Laplacian on a 3 -sphere $S^{3}$. The space of functions on $S^{3}$ carries an action of $\mathrm{so}(4) \cong \mathrm{sl}(2) \oplus \operatorname{sl}(2)$. Therefore, eigenfunctions of the Laplacian on $S^{3}$ are organized in finite dimensional multiplets of $\operatorname{sl}(2) \oplus \operatorname{sl}(2)$. According to the Peter-Weyl theory for $\mathrm{SU}(2) \cong S^{3}$, there is one such multiplet $\varphi_{m}$ for each integer 
$m=0,1,2, \ldots$ It has dimension $d_{m}=(m+1)^{2}$ and transforms in the representation $\left(\frac{m}{2}, \frac{m}{2}\right)$. The eigenvalue of the Laplacian on the multiplet $\varphi_{m}$ is given by $\Delta_{m}=m(m+2)$. For the supersphere $S^{3 \mid 2}$ we expect very similar results except that the multiplicities should roughly exceed those of the bosonic model by a factor of 4 .

Before we extend these thoughts to the supersphere, however, let us mention a few facts on the Lie superalgebra osp(4|2). Its bosonic subalgebra is 9-dimensional and it consists of three commuting copies of $\operatorname{sl}(2)$. This implies that irreducible representations $\left[j_{1}, j_{2}, j_{3}\right]$ of $\operatorname{osp}(4 \mid 2)$ are labeled by three spins $j_{i}$. In these representations the quadratic Casimir element takes the value

$$
C\left(\left[j_{1}, j_{2}, j_{3}\right]\right)=-4 j_{1}\left(j_{1}-1\right)+2 j_{2}\left(j_{2}+1\right)+2 j_{3}\left(j_{3}+1\right) .
$$

A generic (typical)2 2 representation possesses dimension

$$
D\left(\left[j_{1}, j_{2}, j_{3}\right]\right)=16\left(2 j_{1}+1\right)\left(2 j_{2}+1\right)\left(2 j_{3}+1\right) .
$$

The representations of $\operatorname{osp}(4 \mid 2)$ that appear in the spectrum of the Laplacian on the supersphere $S^{3 \mid 2}$ are not generic. On the supersphere, wave functions are organized in $\operatorname{osp}(4 \mid 2)$ multiplets $\phi_{m}, m=0,1,2, \ldots$ The first multiplet $\phi_{0}$ consists of a single function, namely the constant $\phi_{0}=1$. It transforms in the trivial 1-dimensional representation $[0,0,0]$. For positive values of $m$, the multiplet $\phi_{m}$ transforms in the irreducible representation $\left[\frac{1}{2}, \frac{m-1}{2}, \frac{m-1}{2}\right]$ of $\operatorname{osp}(4 \mid 2)$. Consequently, the space $\mathcal{H}_{0}$ of square integrable functions on the supersphere $S^{3 \mid 2}$ decomposes as follows,

$$
\mathcal{H}_{0} \cong[0,0,0] \oplus \bigoplus_{m=1}^{\infty}\left[\frac{1}{2}, \frac{m-1}{2}, \frac{m-1}{2}\right]=\bigoplus_{m=0}^{\infty} \lambda_{m, 0} .
$$

Here we have also introduced the symbol $\lambda_{m, 0}$ such that $\lambda_{0,0}$ is the trivial representation and $\lambda_{m+1,0}=\left[\frac{1}{2}, \frac{m}{2}, \frac{m}{2}\right]$. According to eq. (2.1), the Laplacian takes the values $\Delta_{m}=m^{2}$. The quadratic dependence on $m$ is similar to the bosonic sphere. On the other hand, the degeneracies are much larger for the supersphere. In fact, upon restriction to the bosonic subalgebra, the eigenspaces of the Laplacian decompose according to

$$
\left.\left[\frac{1}{2}, \frac{k}{2}, \frac{k}{2}\right]\right|_{\mathrm{sl}(2) \oplus \mathrm{sl}(2) \oplus \mathrm{sl}(2)} \cong\left(\frac{1}{2}, \frac{k}{2}, \frac{k}{2}\right) \oplus\left(0, \frac{k+1}{2}, \frac{k+1}{2}\right) \oplus\left(0, \frac{k-1}{2}, \frac{k-1}{2}\right)
$$

for $k=m-1 \geq 1$. When $k=0$, the last term must be omitted. The formula implies that the dimension $D_{k}$ of the representation $\lambda_{k, 0}$ is given by $D_{k}=4 k^{2}+2$ for $k \geq 1$. This is roughly four times as large as the dimension of the eigenspaces on the bosonic sphere $S^{3}$, as one would expect.

It is quite instructive to prove the decomposition (2.3). To this end, let us collect the bosonic coordinate functions $x_{i}=: X_{i}, i=1, \ldots, 4$ and the fermionic generators $\eta_{i}=X_{4+i}$

\footnotetext{
${ }^{2}$ See Appendix A.
} 
into a single multiplet $X$. We recall that the six functions $X_{i}$ are subject to the constraint (1.1). The latter may be recast into the more covariant form $X_{a} X_{b} J^{a b}=R^{2}$ by introducing an appropriate matrix $J=\left(J^{a b}\right)$. The multiplet $X$ transforms in the fundamental representation $\lambda_{1,0}=\left[\frac{1}{2}, 0,0\right]$ of $\operatorname{osp}(4 \mid 2)$. When we restrict from $\operatorname{osp}(4 \mid 2)$ to its bosonic subalgebra, $X$ splits into a 4-dimensional multiplet in the $\left(\frac{1}{2}, \frac{1}{2}\right)$ representation of $\mathrm{so}(4) \cong$ $\operatorname{sl}(2) \oplus \operatorname{sl}(2)$ and a 2 -dimensional multiplet in the $\left(\frac{1}{2}\right)$ representation of $\operatorname{sp}(2) \cong \operatorname{sl}(2)$. While the former is spanned by the bosonic coordinate functions $x_{i}$, the latter consists of the odd elements $\eta_{i}$. The algebra $\mathcal{H}_{0}$ of functions on $S^{3 \mid 2}$ is generated by the six coordinates $X_{i}$, i.e. every square integrable function can be arbitrarily well approximated by a polynomial in $X_{i}$. The space of polynomials comes with an integer grading given by the degree of homogeneity. Since the homogeneous polynomials transform in the graded symmetric tensor product of the fundamental representation $\lambda_{1,0}$, one might be inclined to identify the direct sum $S \lambda_{1,0}=\bigoplus \lambda_{1,0}^{\otimes_{s}}$ of all graded symmetric tensor powers of the fundamental representation with the space $\mathcal{H}_{0}$. Such an identification, however, would disregard the defining equation (1.1) of the supersphere. The constraint (1.1) generates an ideal in the symmetric tensor algebra $S \lambda_{1,0}$ that has to be divided out in order to avoid overcounting of states. The twofold symmetric tensor power of the fundamental representation, for example, is given by $\lambda_{1,0}^{\otimes_{s} 2}=[0,0,0] \oplus \lambda_{2,0}$. The constraint (1.1) identifies the multiplet $[0,0,0]$ with the constant function. The latter has been counted already by the very first term $\lambda_{1,0}^{\otimes_{s} 0}=[0,0,0]$. Consequently, when considering the space of homogeneous polynomials in $X_{i}$ up to degree $m$, we have to quotient out the subspace of polynomials that contain the factor $X_{a} X_{b} J^{a b}$, which is isomorphic to the space of homogeneous polynomials of degree less or equal to $m-2$. Thereby we are led to the following expression for $\mathcal{H}_{0}$,

$$
\mathcal{H}_{0}=\lim _{N \rightarrow \infty}\left(\bigoplus_{m=0}^{N} \lambda_{1,0}^{\otimes_{s} m}\right) /\left(\bigoplus_{m=0}^{N-2} \lambda_{1,0}^{\otimes_{s} m}\right)=\bigoplus_{m=0}^{\infty} \lambda_{m, 0}=[0,0,0] \oplus \bigoplus_{k=0}^{\infty}\left[\frac{1}{2}, \frac{k}{2}, \frac{k}{2}\right]
$$

where we have used the tensor product decomposition $3 \lambda_{1,0}^{\otimes_{s} m} \cong \bigoplus_{i=0}^{[m / 2]} \lambda_{m-2 i, 0}$ and the identity $\lambda_{k+1,0}=\left[\frac{1}{2}, \frac{k}{2}, \frac{k}{2}\right]$ for $k \geq 0$.

Before we conclude this subsection, let us briefly construct the partition function for a particle on the supersphere. By this we mean the quantity

$$
Z_{0}=Z_{0}\left(z_{1}, z_{2}, z_{3}\right)=\operatorname{tr}_{\mathcal{H}_{0}}\left(z_{1}^{H^{1}} z_{2}^{H^{2}} z_{3}^{H^{3}}\right)
$$

where $H^{i}$ are the three Cartan generators and the trace is taken evaluated in the space $\mathcal{H}_{0}$ of square integrable functions on the supersphere $S^{3 \mid 2}$. The results we sketched in the previous paragraphs imply that

$$
Z_{0}=1+\sum_{m=0}^{\infty} \chi_{\left[\frac{1}{2}, \frac{m}{2}, \frac{m}{2}\right]}\left(z_{1}, z_{2}, z_{3}\right)
$$

where $\quad \chi_{\left[\frac{1}{2}, \frac{m}{2}, \frac{m}{2}\right]}\left(z_{1}, z_{2}, z_{3}\right)=\chi_{\left(\frac{1}{2}, \frac{m}{2}, \frac{m}{2}\right)}+\chi_{\left(0, \frac{m+1}{2}, \frac{m+1}{2}\right)}+\chi_{\left(0, \frac{m-1}{2}, \frac{m-1}{2}\right)}$.

\footnotetext{
${ }^{3}$ By $[x]$ we mean the floor function of $x$.
} 
In the second line the last term should be omitted for $m=0$ and the character $\chi_{\left(j_{1}, j_{2}, j_{3}\right)}=$ $\prod_{i} \chi_{j_{i}}\left(z_{i}\right)$ denotes a product of bosonic sl(2) characters. The partition function $Z_{0}$ can be written in a different form that mimics our proof of the formula (2.3). To this end, let us consider the module $S \lambda_{1,0}$. We think of it as being generated by four bosonic coordinates in the $\left(\frac{1}{2}, \frac{1}{2}\right)$ representation of $\operatorname{sl}(2) \oplus \operatorname{sl}(2) \cong \mathrm{so}(4)$ along with the two fermionic ones in the $\left(\frac{1}{2}\right)$ representation of $\operatorname{sl}(2) \cong \operatorname{sp}(2)$. On $S \lambda_{1,0}$ we introduce the number operator $N$ that counts the number of bosonic and fermionic coordinate functions in a given monomial. Since there are no non-trivial relations in $S \lambda_{1,0}$ we can easily compute

$$
Z^{S}(t)=\operatorname{tr}_{S \lambda_{1,0}}\left(t^{N} z_{1}^{H^{1}} z_{2}^{H^{2}} z_{3}^{H^{3}}\right)=\frac{\left(1+z_{1}^{\frac{1}{2}} t\right)\left(1+z_{1}^{-\frac{1}{2}} t\right)}{\left(1-z_{2}^{\frac{1}{2}} z_{3}^{\frac{1}{2}} t\right)\left(1-z_{2}^{\frac{1}{2}} z_{3}^{-\frac{1}{2}} t\right)\left(1-z_{2}^{-\frac{1}{2}} z_{3}^{\frac{1}{2}} t\right)\left(1-z_{2}^{-\frac{1}{2}} z_{3}^{-\frac{1}{2}} t\right)}
$$

Multiplying this quantity with $\left(1-t^{2}\right)$ implements the constraint (1.1) on the level of generating functions. We can then remove $t$ by sending it to $t \rightarrow 1$. The result is a rather elegant new formula for the partition function $Z_{0}$,

$$
Z_{0}\left(z_{1}, z_{2}, z_{3}\right)=\lim _{t \rightarrow 1}\left[\left(1-t^{2}\right) Z^{S}\left(t ; z_{1}, z_{2}, z_{3}\right)\right] .
$$

If the quotient is expanded in a Taylor series and expressions are reorganized into characters of $\operatorname{osp}(4 \mid 2)$ we recover our previous result (2.5).

\subsection{The complete boundary spectrum}

Now let us turn to the spectrum of the PCM (1.4) at the special point $R=\infty$ where our field theory becomes free. At this point, the fields are easy to list and their weights agree with their classical values. For simplicity, we shall study the boundary spectrum of a volume filling brane, i.e. with Neumann boundary conditions imposed on all fields of the model. In this case it suffices to consider the derivative $\partial_{u}$ along the boundary, rather than two world-sheet derivatives $\partial$ and $\bar{\partial}$. From now on, the letters $x_{i}=x_{i}(u), \eta_{a}=\eta_{a}(u)$ and $X_{i}=X_{i}(u)$ shall denote boundary fields rather than coordinate functions.

So, let us begin to analyze the space $\mathcal{H}$ of boundary fields. Obviously, $\mathcal{H}$ is spanned by monomials $\Phi$ of the form

$$
\Phi=\prod_{i_{0}} X_{i_{0}} \prod_{i_{1}} \partial X_{i_{1}} \prod_{i_{2}} \partial^{2} X_{i_{2}} \cdots
$$

The number of factors involving no, one, two etc. derivatives $\partial=\partial_{u}$ of the fundamental fields is arbitrary. Let us stress at this point already that the defining relation (1.1) of the supersphere imposes many relations between monomials of the form (2.8). The space $\mathcal{H}$, comes equipped with an integer grading, i.e. $\mathcal{H}=\bigoplus_{n=0}^{\infty} \mathcal{H}_{n}$, where $\mathcal{H}_{n}$ is spanned by monomials $\Phi$ with a total number $n$ of derivatives. The expression $X_{a} \partial X_{b} \partial^{4} X_{c}$, for example, is an element of $\mathcal{H}_{5}$. 
Associated with the integer grading of the state space $\mathcal{H}$ there is a corresponding decomposition of the partition function

$$
Z(q)=\operatorname{str}_{\mathcal{H}}\left(q^{L_{0}-\frac{c}{24}} z_{1}^{H^{1}} z_{2}^{H^{2}} z_{3}^{H^{3}}\right)=q^{-\frac{1}{24}} \sum_{n=0}^{\infty} Z_{n} q^{n}
$$

The coefficients $Z_{n}=Z_{n}\left(z_{i}\right)$ are (infinite) linear combinations of $\operatorname{osp}(4 \mid 2)$ characters. A formula for $Z_{0}$ was discussed in the previous subsection. In the present context it encodes all information on the $\operatorname{osp}(4 \mid 2)$ transformation law of fields with conformal weight $h=0$. These are in one-to-one correspondence with functions on the supersphere $S^{3 \mid 2}$ (recall that we are working at $R=\infty$ ).

Let us now turn to states involving a single derivative $\partial$. Since $\mathcal{H}_{1}$ is built from fields of the form $\phi_{n}\left(X_{i}\right) \partial X_{i}$, where $\phi_{n} \in \mathcal{H}_{0}$, one might at first sight suspect that $Z_{1}^{\prime}=Z_{0} \chi_{\lambda_{1,0}}$ coincides with $Z_{1}$. But this is not true since it actually counts many fields twice. So far, we have not accounted for the derivative of the supersphere relation (1.1). Taking the derivative of this constraint we find

$$
\sum_{i, j} X_{i} \partial X_{j} J^{i j}=0
$$

This additional condition tells us to subtract $Z_{0}$ from $Z_{1}^{\prime}$. Hence we find that $Z_{1}=Z_{0}\left(\chi_{\lambda_{1,0}}-\right.$ $\left.\chi_{\lambda_{0,0}}\right)$ and a simple computer program can decompose this product into characters of osp $(4 \mid 2)$, leading to

$$
Z_{1}=\sum_{k=0}^{\infty}\left(\chi_{\left[1, \frac{k}{2}, \frac{k}{2}\right]}+\chi_{\left[\frac{1}{2}, \frac{k}{2}, \frac{k}{2}\right]}\right)
$$

In order to gain some more familiarity with the state counting we invite the reader to construct the contribution $Z_{2}$ of fields with two derivatives to the total partition function. The answer is given by

$$
\begin{aligned}
Z_{2} & =\chi_{[0,0,0]}+2 \sum_{k=0}^{\infty} \chi_{\left[\frac{1}{2}, \frac{k}{2}, \frac{k}{2}\right]}+\chi_{[1,0,0]} \\
& +\sum_{k=1}^{\infty}\left(\chi_{\left[1, \frac{k+1}{2}, \frac{k-1}{2}\right]}+\chi_{\left[1, \frac{k-1}{2}, \frac{k+1}{2}\right]}+2 \chi_{\left[\frac{1}{2}, \frac{k}{2}, \frac{k}{2}\right]}+2 \chi_{\left[1, \frac{k}{2}, \frac{k}{2}\right]}\right) .
\end{aligned}
$$

Instead of explaining this formula we shall turn to the higher subtraces $Z_{i}$ right away. To begin with, let us enumerate expressions in which no field appears without derivative and where the total degree of the derivatives adds up to $n$. There are $p(n)$ of these terms, where $p(n)$ is the number of partitions of the integer $n$. We shall denote the set of partitions by $P(n)$ and think of their elements as sequences $\mu=\left(\mu_{i}, i=1,2,3, \ldots\right)$ such that $\sum i \mu_{i}=n$. With $n=3$, for example, we have to consider terms involving $\partial^{3} X_{i}, \partial^{2} X_{i} \partial X_{j}$ and $\partial X_{i} \partial X_{j} \partial X_{k}$ corresponding to the sequences $\left(\mu_{1}, \mu_{2}, \mu_{3}\right)=(0,0,1),(1,1,0)$ and $(3,0,0)$, respectively. In our notations we shall suppress the infinite number of zero entries to the right of the last non-zero one. To each partition $\mu \in P(n)$, we associate the trace $\chi_{\lambda_{1,0}^{\otimes \mu}}$ over the space 
$\lambda_{1,0}^{\otimes_{s} \mu_{1}} \otimes \lambda_{1,0}^{\otimes_{s} \mu_{2}} \cdots$,

$$
\chi_{\lambda_{1,0}^{\otimes \mu}}\left(z_{1}, z_{2}, z_{3}\right)=\prod_{i=1}^{\infty} \chi_{\lambda_{1,0}^{\otimes_{s} \mu_{i}}}
$$

The factors on the right hand side involve traces over the $\mu_{i}^{\text {th }}$ symmetric tensor product of the fundamental representation $\lambda_{1,0}$. Such factors arise from the product of $\mu_{i}$ derivatives of order $i$ of the fundamental field multiplet. Let us now set $Z_{n}^{\prime}=Z_{0} \sum_{\mu \in P(n)} \chi_{\lambda_{1,0}^{\otimes \mu}}$ to be $Z_{0}$ multiplied with the sum of the $p(n)$ traces (2.12). Clearly, $Z_{n}^{\prime}$ is not the same as $Z_{n}$. In fact, we still have to correct for some overcounting, since we have to subtract all possible derivatives of degree up to $n$ of the supersphere relations (1.1). Each one of the $p(n)$ partitions $\mu \in P(n)$ has to be investigated on its own in order to understand which relations apply to it. Suppose that for a given partition $\mu$, the entry $\mu_{j}$ does not vanish. This means that the corresponding fields contain a factor $\partial^{j} X_{a}$. Hence, there exist relations between such fields that arise from the $j^{\text {th }}$ derivative of the supersphere relation (1.1). These must be removed. We may formalize this prescription by introducing the special partitions $\epsilon^{i}$ which have a single entry $\epsilon_{i}^{i}=1$ in the $i^{\text {th }}$ position and are zero otherwise. The sequence $\epsilon^{i}$ is an element of $P(i)$. Let us also denote by $\mu-\epsilon^{i}$ the partition from $P(n-i)$ that is obtained by subtracting the entries. If the resulting sequence contains a negative entry, i.e. if $\mu_{i}=0$, then we set $\chi_{\lambda_{1,0}^{\otimes\left(\mu-\epsilon^{i}\right)}}=0$. With these notations, we can now formalize our resolution for the issue of overcounting. Taking into account the constraints imposed by the $i^{\text {th }}$ derivative of (1.1) amounts to subtracting from $Z_{n}^{\prime}$ all functions of the form $Z_{0} \chi_{\lambda_{1,0}^{\otimes\left(\mu-\epsilon^{i}\right)}}$. Here, $\mu \in P(n)$ and $i$ runs through all integers $i=1,2, \ldots$ such that $\mu_{i} \neq 0$. After removing all these terms from $Z_{n}^{\prime}$ we realize that we actually overdid things with our correction. In fact we have deleted those expressions for which two ore more relations are simultaneously fulfilled, so that we need to put them back in. Thus, we must add all the terms $Z_{0} \chi_{\lambda_{1,0}^{\otimes\left(\mu-\epsilon^{i}-\epsilon^{j}\right)}}$ with $i<j$. The resulting expression overcounts those polynomials that obey three different relations, etc. A simple induction leads to the following expression for $Z_{n}$

$$
Z_{n}=Z_{0} \sum_{\mu \in P(n)}\left(\chi_{\left[\frac{1}{2}, 0,0\right] \otimes \mu}-\sum_{i=1}^{n} \chi_{\left[\frac{1}{2}, 0,0\right] \otimes\left(\mu-\epsilon^{i}\right)}+\sum_{i<j=1}^{n} \chi_{\left[\frac{1}{2}, 0,0\right] \otimes\left(\mu-\epsilon^{i}-\epsilon^{j}\right)}-\cdots\right) .
$$

All notations that are used in this expression have been introduced in the preceding paragraph. We have placed the subscript $\lambda_{1,0}=\left[\frac{1}{2}, 0,0\right]$ back on the symbol $\chi$ to emphasize the relation to the fundamental multiplet. The reader is invited to check that our general formula for $Z_{n}$ reproduces the previous expressions (2.5/2.10|2.11) for $Z_{n}$ when $n \leq 2$.

Having found a formula for $Z_{n}$, we can insert it into our general prescription (2.9). The result is,

$Z=q^{-\frac{1}{24}} Z_{0} \sum_{n=0}^{\infty} q^{n} \sum_{\mu \in P(n)}\left(\chi_{\left[\frac{1}{2}, 0,0\right] \otimes \mu}-\sum_{i=1}^{n} \chi_{\left[\frac{1}{2}, 0,0\right] \otimes\left(\mu-\epsilon^{i}\right)}+\sum_{i<j=1}^{n} \chi_{\left[\frac{1}{2}, 0,0\right] \otimes\left(\mu-\epsilon^{i}-\epsilon^{j}\right)}-\cdots\right)$.

Now, since $\mu-\epsilon^{j}$ is a partition in $P(n-j)$, we are led to the idea of combining in the above alternating sum all those terms that belong to partitions of the same size. Denoting by 
$p_{d}(x ; y)$ the function that counts the number of distinct, i.e. whose elements are all different, partitions of $x$ with exactly $y$ elements, we leave to the reader the combinatorial homework to deduce

$$
\begin{aligned}
& Z=q^{-\frac{1}{24}} Z_{0} \sum_{n=0}^{\infty} q^{n}(\sum_{j=0}^{n} \underbrace{\left(\sum_{k=0}^{j}(-1)^{k} p_{d}(j ; k)\right)}_{=: c_{j}} \sum_{\mu \in P(n-j)} \chi_{\left[\frac{1}{2}, 0,0\right] \otimes \mu}) \\
= & q^{-\frac{1}{24}} Z_{0} \sum_{n, j=0}^{\infty} q^{n} c_{j} \sum_{\mu \in P(n-j)} \chi_{\left[\frac{1}{2}, 0,0\right] \otimes \mu}=q^{-\frac{1}{24}} Z_{0}\left(\sum_{j=0}^{\infty} c_{j} q^{j}\right) \sum_{n=0}^{\infty} q^{n} \sum_{\mu \in P(n)} \chi_{\left[\frac{1}{2}, 0,0\right] \otimes \mu} \\
= & q^{-\frac{1}{24}} Z_{0} \phi(q) \sum_{n=0}^{\infty} q^{n} \sum_{\mu \in P(n)} \chi_{\left[\frac{1}{2}, 0,0\right] \otimes \mu} .
\end{aligned}
$$

The numbers $c_{j}$ can easily be recognized as the coefficients in the Taylor expansion of the Euler $\phi$-function. In fact the generating function for distinct partitions of a number $n$ into precisely $l$ distinct numbers is given by

$$
\prod_{k=1}^{\infty}\left(1+z q^{k}\right)=\sum_{n=0}^{\infty} \sum_{l=0}^{n} p_{d}(n ; l) z^{l} q^{n} .
$$

For $z=-1$ the left hand side reduces to the Euler function $\phi(q)$ while the right hand side gives the $\operatorname{sum} \sum_{n=0}^{\infty} c_{n} q^{n}$. Note that during the resummation in the second line of eq. (2.14) we could drop a number of terms since $P(n)$ is empty for $n<0$. The result (2.14) has a rather surprising interpretation. It tells us that we may at first discard all the derivatives of the supersphere relations for the computation of subtraces $Z_{i}$. Derivatives of eq. (1.1) may then simply be taken into account by multiplying the result with the Euler function $\phi(q)$.

The conclusion of the previous discussion may now be employed to derive a much simpler formula for the partition function which generalizes the expression (2.7) for $Z_{0}$. Without paying respect to the supersphere relations, it is straightforward to enumerate derivative fields. Recall that the four fundamental bosonic fields carry charges $\left(0, \pm \frac{1}{2}, \pm \frac{1}{2}\right)$ under the three Cartan generators $\left(H^{1}, H^{2}, H^{3}\right)$. Similarly, the two fundamental fermionic fields are only charged under the first Cartan generator $H^{1}$ such that their charges are $\left( \pm \frac{1}{2}, 0,0\right)$. Hence, the partition function can now be represented in the form

$$
Z=q^{-\frac{1}{24}} Z_{0} \phi(q) \prod_{n=1}^{\infty} \frac{\left(1+z_{1}^{\frac{1}{2}} q^{n}\right)\left(1+z_{1}^{-\frac{1}{2}} q^{n}\right)}{\left(1-z_{2}^{\frac{1}{2}} z_{3}^{\frac{1}{2}} q^{n}\right)\left(1-z_{2}^{\frac{1}{2}} z_{3}^{-\frac{1}{2}} q^{n}\right)\left(1-z_{2}^{-\frac{1}{2}} z_{3}^{\frac{1}{2}} q^{n}\right)\left(1-z_{2}^{-\frac{1}{2}} z_{3}^{-\frac{1}{2}} q^{n}\right)} .
$$

The infinite product enumerates all states in the unconstrained state space. According to our previous discussion, the derivatives of the supersphere constraints can be implemented through a simple multiplication with the Euler function $\phi(q)$. Our final formula for the partition function of a volume filling brane in the PCM at $R=\infty$ is indeed very simple. 


\subsection{Casimir decomposition of the boundary spectrum}

The goal of this section is to expand the partition sum (2.9) of the volume filling brane in terms of $\operatorname{osp}(4 \mid 2)$ characters. To be more concrete, we would like to derive explicit formulas for the branching functions $\psi_{\Lambda}^{K}(q)$ in the decomposition

$$
Z\left(q, z_{1}, z_{2}, z_{3}\right)=\sum_{\Lambda} \chi_{\Lambda}^{K}\left(z_{1}, z_{2}, z_{3}\right) \psi_{\Lambda}^{K}(q)
$$

Here, the functions $\chi_{\Lambda}^{K}\left(z_{1}, z_{2}, z_{3}\right)$ are characters of the Kac module $4 K_{\Lambda}$ of osp $(4 \mid 2)$. The latter form a basis in the space of all characters so that the expansion coefficients are uniquely determined. Finding an explicit formula for the branching functions $\psi_{\Lambda}^{K}(q)$ is the main result of this section. The final expression will take the following form

$$
\begin{array}{r}
\psi_{\left[j_{1}, j_{2}, j_{3}\right]}^{K}(q)=\frac{q^{2 j_{1}\left(j_{1}-1\right)}-j_{2}\left(j_{2}+1\right)-j_{3}\left(j_{3}+1\right)}{\eta(q) \phi(q)^{3}} \sum_{n, m=0}^{\infty}(-1)^{m+n} q^{\frac{m}{2}\left(m+4 j_{1}+2 n+1\right)+\frac{n}{2}+j_{1}} \\
\times\left(q^{\left(j_{2}-\frac{n}{2}\right)^{2}}-q^{\left(j_{2}+\frac{n}{2}+1\right)^{2}}\right)\left(q^{\left(j_{3}-\frac{n}{2}\right)^{2}}-q^{\left(j_{3}+\frac{n}{2}+1\right)^{2}}\right) .
\end{array}
$$

Let us add two remarks here. To begin with, the decomposition (2.17) of the supersphere partition function has also been considered in the work of Candu and Saleur [23, 24]. In their context, the branching functions $\psi^{K}$ are related to representation spaces of the so-called Brauer algebra. The connection has interesting implications, but it does not provide explicit formulas for $\psi^{K}$. Our formula (2.18) has not appeared in the literature before. In addition, we would want to stress that the decomposition of the partition function into characters of Kac modules is a somewhat formal procedure that does not fully capture the representation content of the spectrum, at least not for the atypical sector of the theory. One may notice, for example, that some of the expansion coefficients $C_{n}$ in $\psi_{\Lambda}^{K}(q)=\sum C_{n} q^{n}$ are negative. Only for typical $\Lambda$ will the $c_{n}=C_{n}^{\Lambda}$ are positive. For atypical representations $\Lambda$, on the other hand, the characters $\chi_{\Lambda}^{K}$ of the Kac modules have to be decomposed into characters of irreducible atypical representations $\chi_{\Lambda}$ as described in (C.11) in order to obtain branching functions with non-negative integral multiplicities.

The proof of eq. (2.18) proceeds in several steps. To begin with, we shall decompose the partition function into representations of the bosonic subalgebra of osp(4|2). Our second step then is to recombine bosonic characters into the characters of full osp(4|2) multiplets. Once this is achieved, the resulting expressions still require some resummation in order to bring them into a more appealing form.

In our computation, we shall split the full partition function into three different parts and decompose them separately before putting all this together. We shall start with the fermionic contributions in the numerator of the partition function (2.16). Apart from the factors that arise from derivative fields, there are also two terms in $Z_{0}$ that account for fermionic zero modes. We may simply set the parameter $t$ to $t=1$ in those two factors and combine them

\footnotetext{
${ }^{4}$ Again, see Appendix A.
} 
with the $q$-dependent terms in the numerators of eq. (2.16) to obtain

$$
\begin{aligned}
Z^{\mathrm{F}}\left(q, z_{1}\right) & :=\prod_{n=0}^{\infty}\left(1+z_{1}^{\frac{1}{2}} q^{n}\right)\left(1+z_{1}^{-\frac{1}{2}} q^{n}\right)=\left(1+z_{1}^{\frac{1}{2}}\right) \prod_{n=0}^{\infty}\left(1+z_{1}^{\frac{1}{2}} q^{n+1}\right)\left(1+z_{1}^{-\frac{1}{2}} q^{n}\right) \\
& =q^{-\frac{1}{8}}\left(z_{1}^{-\frac{1}{4}}+z_{1}^{\frac{1}{4}}\right) \frac{1}{\phi(q)} \theta_{2}\left(z_{1}^{\frac{1}{2}} \mid q\right)=\frac{1}{\phi(q)} \sum_{n \in \mathbb{Z}} z_{1}^{\frac{n}{2}}\left(q^{\frac{n(n+1)}{2}}+q^{\frac{n(n-1)}{2}}\right) \\
& =\frac{1}{\phi(q)} \sum_{n=0, \frac{1}{2}, 1, \ldots}\left(q^{n(2 n+1)}+q^{n(2 n-1)}-q^{(n+1)(2 n+3)}-q^{(n+1)(2 n+1)}\right) \chi_{n}\left(z_{1}\right) .
\end{aligned}
$$

Along the way we have used a number of simple identities 5 for $\theta$-functions. As a result, all the fermionic contributions to the partition function have been decomposed explicitly into multiplets of the even part of $\operatorname{osp}(4 \mid 2)$. Note that the two fermions transform non-trivially only under the first subalgebra $\operatorname{sl}(2)$ and hence there is no dependence on $z_{2}$ and $z_{3}$ this time.

The second piece of the partition function (2.16) that we would like to split off concerns the bosonic zero modes, i.e. the denominator of the minisuperspace partition function $Z_{0}$. Its decomposition into bosonic representations is straightforward

$$
\lim _{t \rightarrow 1} \frac{1-t^{2}}{\left(1-z_{2}^{\frac{1}{2}} z_{3}^{\frac{1}{2}} t\right)\left(1-z_{2}^{\frac{1}{2}} z_{3}^{-\frac{1}{2}} t\right)\left(1-z_{2}^{-\frac{1}{2}} z_{3}^{\frac{1}{2}} t\right)\left(1-z_{2}^{-\frac{1}{2}} z_{3}^{-\frac{1}{2}} t\right)}=\sum_{n=0, \frac{1}{2}, 1, \ldots} \chi_{n}\left(z_{2}\right) \chi_{n}\left(z_{3}\right)
$$

Note that the sum of characters on the left hand side encodes the well-known spectrum of a bosonic 3 -sphere $S^{3} \cong \mathrm{SU}(2)$. Therefore we can just state this equality without any detailed calculation. The commuting left and right invariant vector fields are generated by the second and third copy of $\operatorname{sl}(2)$ within the even part of osp(4|2). Hence, there is no dependence on the parameter $z_{1}$.

It remains to analyze the $q$-dependent factors in the denominator of the partition function (2.16). Their contribution may be expanded as follows

$$
\begin{aligned}
\prod_{n=1}^{\infty} & \left(\left(1-z_{2}^{\frac{1}{2}} z_{3}^{\frac{1}{2}} q^{n}\right)\left(1-z_{2}^{\frac{1}{2}} z_{3}^{-\frac{1}{2}} q^{n}\right)\left(1-z_{2}^{-\frac{1}{2}} z_{3}^{\frac{1}{2}} q^{n}\right)\left(1-z_{2}^{-\frac{1}{2}} z_{3}^{-\frac{1}{2}} q^{n}\right)\right)^{-1} \\
& =\left(\sum_{n \in \mathbb{Z}} \frac{z_{2}^{\frac{n}{2}} z_{3}^{\frac{n}{2}}}{\phi(q)^{2}} \sum_{m=0}^{\infty}(-1)^{m}\left(q^{\frac{m}{2}(m+2 n+1)}-q^{\frac{m}{2}(m+2 n-1)}\right)\right) \times\left(z_{3} \longrightarrow z_{3}^{-1}\right) \\
& =\sum_{\substack{k, l \in \mathbb{Z} \\
k+l \in 2 \mathbb{Z}}} \frac{z_{2}^{\frac{k}{2}} z_{3}^{\frac{l}{2}}}{\phi(q)^{4}} \sum_{n, m=1}^{\infty}(-1)^{n+m} q^{\frac{n+m}{2}+l \frac{n-m}{2}}\left(q^{\frac{n(n+1)}{2}}-q^{\frac{n(n-1)}{2}}\right)\left(q^{\frac{m(m+1)}{2}}-q^{\frac{m(m-1)}{2}}\right) \\
& =\frac{1}{\phi(q)^{4}} \sum_{\substack{k, l \in \mathbb{N} \\
k+l \in 2 \mathbb{N}}} \sum_{n, m=1}^{\infty}(-1)^{n+m} \frac{\left(1-q^{n}\right)\left(1-q^{m}\right)\left(1-q^{n+m}\right)\left(1-q^{n-m}\right)}{q^{-(k(n+m)+l(n-m)+n(n-1)+m(m-1)) / 2}} \chi_{\frac{k}{2}}\left(z_{2}\right) \chi_{\frac{l}{2}}\left(z_{3}\right) .
\end{aligned}
$$

\footnotetext{
${ }^{5}$ See equation (B.1).
} 
In the first line of the above computation we have used the lemma (B.1). Since all the contributions being captured by this computation are associated with bosonic fields, characters with a non-trivial $z_{1}$ dependence do not arise.

In order to obtain the decomposition of $Z$ into characters of osp $(4 \mid 2)_{\overline{0}} \cong \operatorname{sl}(2) \oplus \operatorname{sl}(2) \oplus \operatorname{sl}(2)$, we need to put the results from the preceding three computations together into one expression. The answer contains products of characters which depend on the same variables $z_{2}$ and $z_{3}$. These products can be re-expanded with the help of the following auxiliary formula

$$
\begin{aligned}
\sum_{p=0}^{\infty} \chi_{\frac{p}{2}}\left(z_{2}\right) \chi_{\frac{p}{2}}\left(z_{3}\right) \sum_{\substack{k, l \in \mathbb{N} \\
k+l \in 2 \mathbb{N}}} a_{k, l} \chi_{\frac{k}{2}}\left(z_{2}\right) \chi_{\frac{l}{2}}\left(z_{3}\right) \\
=\sum_{\substack{k, l \in \mathbb{N} \\
k+l \in 2 \mathbb{N}}} \chi_{\frac{k}{2}}\left(z_{2}\right) \chi_{\frac{l}{2}}\left(z_{3}\right)\left(\sum_{p=0}^{\infty} \sum_{r=0}^{\min \{k, p\}} \sum_{s=0}^{\min \{l, p\}} a_{|k-p|+2 r,|l-p|+2 s}\right)
\end{aligned}
$$

which holds for an arbitrary set of numbers $a_{k, l}$. When applied to the case at hand, we find

$$
\begin{aligned}
Z=\frac{1}{\phi(q)^{3} \eta(q)} Z^{\mathrm{F}}\left(q, z_{1}\right) & \sum_{\substack{j_{2}, j_{3} \in \frac{1}{2} \mathbb{N} \\
j_{2}+j_{3} \in \mathbb{N}}} \chi_{j_{2}}\left(z_{2}\right) \chi_{j_{3}}\left(z_{3}\right) \sum_{m, n=1}^{\infty}(-1)^{m+n} q^{\frac{n(n-1)}{2}+\frac{m(m-1)}{2}} \\
& \times\left(1-q^{n+m}\right)\left(q^{(n-m)\left(j_{2}-j_{3}\right)}-q^{(n-m)\left(j_{2}+j_{3}+1\right)}\right)
\end{aligned}
$$

Thereby, we completed out first task, namely to decompose the full partition function $Z$ into irreducible representations of the bosonic subalgebra of $\operatorname{osp}(4 \mid 2)$.

Our next issue is to combine bosonic characters back into the characters of Kac modules of $\operatorname{osp}(4 \mid 2)$. Since the even part of $\operatorname{osp}(4 \mid 2)$ is a subalgebra of $\operatorname{osp}(4 \mid 2)$, it is clear that the characters of osp $(4 \mid 2)$ Kac modules, possess a decomposition into characters of the bosonic subalgebra. These decomposition formulas may be inverted such that bosonic characters can be written as infinite linear combinations of osp $(4 \mid 2)$ characters. All necessary details are provided in Appendix C. The resulting expression for the partition function $Z$ is of the form (2.17) with

$$
\begin{aligned}
\psi_{\left[j_{1}, j_{2}, j_{3}\right]}^{K}(q)= & \frac{1}{\eta(q) \phi(q)^{3}} \sum_{k=0}^{\infty} \sum_{m, n=1}^{\infty} \sum_{l=0}^{\infty}(-1)^{m+n+k} q^{2 j_{1}\left(j_{1}+k+2 l\right)} q^{\frac{n(n-1)}{2}+\frac{m(m-1)}{2}} \\
& \times \sum_{r, s=0}^{k} q^{(n-m)(r-s)}\left(1-q^{n+m}\right)\left(q^{(n-m)\left(j_{2}-j_{3}\right)}-q^{(n-m)\left(j_{2}+j_{3}+1\right)}\right) \\
\times & {\left[q^{j_{1}+\frac{k+2 l}{2}(k+2 l+1)}+q^{-j_{1}+\frac{k+2 l}{2}(k+2 l-1)}-q^{5 j_{1}+3+\frac{k+2 l}{2}(k+2 l+5)}-q^{3 j_{1}+\frac{k+2 l}{2}(k+2 l+3)}\right] } \\
= & \frac{q^{2 j_{1}\left(j_{1}-1\right)}}{\eta(q) \phi(q)^{3}} \sum_{m, n=1}^{\infty} \sum_{k=-\infty}^{\infty}(-1)^{k} \sum_{l=0}^{\infty} q^{j_{1}(2|k|+4 l+1)+\frac{|k|}{2}(|k|-1)+l(2 l+2|k|-1)}\left(1-q^{|k|+2 l+2 j_{1}}\right) \\
& \times(-1)^{m+n} q^{\frac{n(n-1)}{2}+\frac{m(m-1)}{2}} q^{(n-m) k}\left(1-q^{n+m}\right)\left(q^{(n-m)\left(j_{2}-j_{3}\right)}-q^{(n-m)\left(j_{2}+j_{3}+1\right)}\right) .
\end{aligned}
$$


We will now make several transformations and resummations in order to cast this unwieldy expression into the form (2.18) we have spelled out above. Making the substitution $n+m=$ $r+2, n-m=s$ with $r \in \mathbb{N}$ and $s=-r,-r+2, \ldots, r$, using the trick (B.2) and then substituting $r \rightarrow r+1$ gives the result

$$
\begin{aligned}
& \psi^{K}(q)=\frac{q^{2 j_{1}\left(j_{1}-1\right)}}{\eta(q) \phi(q)^{3}} \sum_{k=-\infty}^{\infty} \sum_{r, l=0}^{\infty}(-1)^{r+k} q^{j_{1}(2|k|+1)+\frac{|k|(|k|-1)}{2}+l\left(2 l+2|k|+4 j_{1}-1\right)}\left(q^{|k|+2 l+2 j_{1}}-1\right) \\
& \times q^{\frac{(r+2)(r+1)}{2}}\left(q^{(r+1)\left(j_{2}-j_{3}+k\right)}+q^{(r+1)\left(-j_{2}+j_{3}-k\right)}-q^{(r+1)\left(j_{2}+j_{3}+1+k\right)}-q^{(r+1)\left(-j_{2}-j_{3}-1-k\right)}\right) .
\end{aligned}
$$

In order to simplify the sum over $r$, we now need to split the summation over $k$ into three parts, according to whether it is positive, zero or negative. We then recombine the summations over positive and negative $k$ into a single sum and employ another auxiliary formula (B.3) from Appendix B to find

$$
\begin{aligned}
& \psi_{\left[j_{1}, j_{2}, j_{3}\right]}^{K}(q)=q^{2 j_{1}\left(j_{1}-1\right)-j_{2}\left(j_{2}+1\right)-j_{3}\left(j_{3}+1\right)} \frac{1}{\eta(q) \phi(q)^{3}} \sum_{l=0}^{\infty} \sum_{r=0}^{\infty}(-1)^{r} q^{\frac{r}{2}+j_{1}} \\
& \quad \times\left(q^{\left(j_{2}-\frac{r}{2}\right)^{2}}-q^{\left(j_{2}+\frac{r}{2}+1\right)^{2}}\right)\left(q^{\left(j_{3}-\frac{r}{2}\right)^{2}}-q^{\left(j_{3}+\frac{r}{2}+1\right)^{2}}\right)\left[q^{l\left(2 l+4 j_{1}-1\right)}\left(1+q^{2 l+2 j_{1}}\right)\right. \\
& \left.\quad+\sum_{k=1}^{\infty}(-1)^{k} q^{j_{1}(2 k+1)+\frac{k(k-1)}{2}+l\left(2 l+2 k+4 j_{1}-1\right)}\left(1-q^{r+1}\right)\left(q^{(r+1)(k-1)}+q^{-(r+1) k}\right)\right] .
\end{aligned}
$$

Once again we need to rearrange the sum over $k$. Terms can be combined into a single summation if we let $l$ run over half-integers rather than integers. Making the substitutions $l \rightarrow 2 m$ and $r \rightarrow n$, leads to the formula

$$
\begin{gathered}
\psi_{\left[j_{1}, j_{2}, j_{3}\right]}^{K}(q)=\frac{q^{2 j_{1}\left(j_{1}-1\right)-j_{2}\left(j_{2}+1\right)-j_{3}\left(j_{3}+1\right)}}{\eta(q) \phi(q)^{3}} \sum_{n, m=0}^{\infty} \sum_{k=-\infty}^{\infty}(-1)^{m+n+k} q^{\frac{m}{2}\left(m+4 j_{1}-1\right)+\frac{n}{2}+j_{1}} \\
\times\left(q^{\left(j_{2}-\frac{n}{2}\right)^{2}}-q^{\left(j_{2}+\frac{n}{2}+1\right)^{2}}\right)\left(q^{\left(j_{3}-\frac{n}{2}\right)^{2}}-q^{\left(j_{3}+\frac{n}{2}+1\right)^{2}}\right) q^{|k|\left(2 j_{1}+m\right)+\frac{|k||| k \mid-1)}{2}+(n+1) k} .
\end{gathered}
$$

It is advantageous to split the summation over $k$ again depending on whether $k$ is negative or non-negative. Then we substitute $r$ for the sum $r=m+k$ and $s$ for the difference $s=m-k$. After some rather trivial but tedious steps we can thereby bring $\psi^{K}$ into the form

$$
\begin{gathered}
\psi_{\left[j_{1}, j_{2}, j_{3}\right]}^{K}(q)=\frac{q^{2 j_{1}\left(j_{1}-1\right)-j_{2}\left(j_{2}+1\right)-j_{3}\left(j_{3}+1\right)}}{\eta(q) \phi(q)^{3}} \sum_{n, m=0}^{\infty}(-1)^{m+n} q^{\frac{m}{2}\left(m+4 j_{1}+2 n+1\right)+\frac{n}{2}+j_{1}} \\
\times\left(q^{\left(j_{2}-\frac{n}{2}\right)^{2}}-q^{\left(j_{2}+\frac{n}{2}+1\right)^{2}}\right)\left(q^{\left(j_{3}-\frac{n}{2}\right)^{2}}-q^{\left(j_{3}+\frac{n}{2}+1\right)^{2}}\right) \sum_{s=0}^{2 m} q^{-s(n+1)}
\end{gathered}
$$

It is left to the reader to use lemma $(\overline{B .2})$ in order to show that this is equal to the formula (2.18) we spelled out at the beginning of this section. Before we conclude our discussion of the large volume limit, let us stress that our decomposition (2.17) does not imply that 
states actually transform in Kac modules of osp $(4 \mid 2)$. The partition sum does not contain any information on how irreducible atypical representations are actually combined into indecomposables of osp $(4 \mid 2)$. For us, the characters of Kac modules were simply a convenient basis to use.

\section{The $\operatorname{OSP}(4 \mid 2)$ GN model and the supersphere $\mathrm{S}^{3 \mid 2}$}

In this section we shall study the conjectured dual GN model. We begin with the free bulk theory defined by eq. (1.5). After a brief discussion of the bulk spectrum for generic $S$ we specialize to $S=1$ and re-express the bulk partition function through characters of the model's affine $\widehat{\mathrm{osp}}(4 \mid 2)$ symmetry at level $k=16$ In section 3.2 we analyze one particular symmetry preserving boundary condition and spell out its spectrum. The latter is then decomposed according to the action of the global osp(4|2) symmetry in the third subsection. Once such a Casimir decomposition has been performed, we can apply the results of [22] and determine the boundary spectrum throughout the entire moduli space that is generated by the deformation. We shall show that at $R=\infty$ we recover precisely the spectrum of the volume filling brane in the PCM on the supersphere $S^{3 \mid 2}$.

\subsection{Free field construction of the bulk theory}

Before we discuss the spectrum and symmetries of the free Gross-Neveu model (1.5), it is useful to recall how things work for the case $S=0$, i.e. for the fermionic description of the free boson. As is well known, the compactified free boson at radius $R=1$ is equivalent to the free field theory of two real fermions. Each of the two fermionic fields gives rise to a copy of the Ising model with $c=1 / 2$. The two factors, however, are coupled by an orbifold construction to ensure that only sectors contribute in which both fermions obey the same (anti-)periodic boundary conditions. In the next few paragraphs we would like to formalize this construction. It will turn out rather useful for the generalization to $S>0$.

Let us begin with a few words on the sectors of the critical Ising model. We recall that the Virasoro algebra with $c=1 / 2$ possesses three sectors which we shall label by the conformal weights of their ground states, i.e. through $[0],[1 / 2]$ and $[\sigma]=[1 / 16]$. The character functions of these sectors read as follows,

$$
\chi_{\epsilon}(q)=\frac{1}{2}\left(\sqrt{\frac{\theta_{3}}{\eta}}+(-1)^{2 \epsilon} \sqrt{\frac{\theta_{4}}{\eta}}\right) \quad, \quad \chi_{\sigma}(q)=\frac{1}{\sqrt{2}} \sqrt{\frac{\theta_{2}}{\eta}}
$$

with the slightly unusual notation $\epsilon=0,1 / 2$. This will turn out rather convenient below. The product of two Ising models contains a special sector $\gamma=[1 / 2,1 / 2]$ with weight $h=1$. It generates an abelian group $\Gamma_{0}=\mathbb{Z}_{2}$ in the fusion ring. Elements of this group are called simple currents since their fusion with an arbitrary representation always yields a single

\footnotetext{
${ }^{6}$ The discrepancy between our value $k=1$ and the $k=-1 / 2$ that appears in the work of Candu and Saleur is entirely due to different conventions.
} 
contribution. We claim that the corresponding simple current orbifold model is equivalent to the compactified free boson at $R=1$.

The construction of a simple current orbifold proceeds in several simple steps. To begin with, we have to list all sectors $[J]$ of the theory which possess integer monodromy charge $Q_{J}(\gamma)=h_{J}+h_{\gamma}-h_{\gamma \times J}$. These are then organized into orbits $\mathcal{O}_{a}$ under the action of the simple current group $\Gamma$. Each such orbit $\mathcal{O}_{a}$ contributes one term $Z_{a}$ to the partition function of the orbifold model, with a coefficient $|\Gamma| /\left|\mathcal{O}_{a}\right|$ that is given by the ratio between the order $|\Gamma|$ of the orbifold group and the length $\left|\mathcal{O}_{a}\right|$ of the orbit (see e.g. [26]). In our case, there exist five sectors $[J]=\left[\epsilon_{1}, \epsilon_{2}\right]$ and $[J]=[\sigma, \sigma]$ with integer monodromy charge. Under the action of $\Gamma_{0}$ they are organized into three orbits, two of length two and one that is left invariant by fusion with $\gamma$. Consequently, the associated simple current orbifold invariant becomes

$$
Z_{\mathrm{Ising}}^{\mathrm{orb}\left(\Gamma_{0}\right)}(q)=Z_{S=0}^{\mathrm{FF}}(q)=\left|\chi_{(0,0)}+\chi_{(1 / 2,1 / 2)}\right|^{2}+\left|\chi_{(0,1 / 2)}+\chi_{(1 / 2,0)}\right|^{2}+2\left|\chi_{(\sigma, \sigma)}\right|^{2} .
$$

The characters on the right hand side are products of characters of the $c=1 / 2$ Virasoro algebra, i.e. $\chi_{(0,1 / 2)}(q)=\chi_{0}(q) \chi_{1 / 2}(q)$ etc. According to the claims we stated above, the simple current orbifold (3.2) agrees with the free boson compactified at radius $R=1$,

$$
Z_{S=0}^{\mathrm{FF}}(q)=\frac{1}{|\eta(q)|^{2}} \sum_{n, w} q^{\frac{1}{8}(n+2 w)^{2}} \bar{q}^{\frac{1}{8}(n-2 w)^{2}}=Z^{R=1}(q) .
$$

The detailed proof of this identity can be found e.g. in the lectures of Ginsparg [27]. Our aim now is to extend eq. (3.3) to the case $S>0$.

For $S>0$, our theory (1.5) is built from $2 S+2$ real fermions whose properties we have reviewed already. In addition there are also $S$ free $\beta \gamma$-systems with central charge $c=-1$ (see [28] for a detailed analysis of this rather unusual CFT in the context of our work). For $\operatorname{osp}(2 S+2 \mid 2 S)$ symmetry it is necessary that all these fields obey the same boundary conditions, i.e. are either all periodic or all anti-periodic. Before we spell out the relevant bulk partition function, we need a bit more background on the $\beta \gamma$-systems.

As in the case of real fermions, we shall consider sectors which differ by the choice of boundary conditions on the fields $\beta$ and $\gamma$. Let us introduce a family of ground states $|\nu\rangle$ for $\nu \in \frac{1}{2} \mathbb{Z}$. These states are characterized by the conditions

$$
\beta_{r+\nu}|\nu\rangle=0 \quad, \quad \gamma_{r-\nu}|\nu\rangle=0 \quad \text { for } \quad r=1 / 2,3 / 2,5 / 2, \ldots
$$

From the ground states we generate the corresponding sectors by application of raising operators. If we assign charges $q_{\beta}=1 / 2$ and $q_{\gamma}=-1 / 2$ to the modes of the fields $\beta$ and $\gamma$, respectively, and $q_{\nu}=\nu / 2$ to the ground state $|\nu\rangle$ the generating function for the sector $\nu$ reads,

$$
\chi^{(\nu)}(q, y)=q^{\frac{1}{24}-\frac{\nu^{2}}{2}} y^{\frac{\nu}{2}} \prod_{n=0}^{\infty} \frac{1}{\left(1-y^{\frac{1}{2}} q^{n+\frac{1}{2}-\nu}\right)\left(1-y^{-\frac{1}{2}} q^{n+\frac{1}{2}+\nu}\right)}=\frac{q^{-\nu^{2} / 2} y^{\frac{\nu}{2}} \eta(q)}{\theta_{4}\left(q, y^{1 / 2} q^{-\nu}\right)}
$$


All the constructed sectors carry an action of an affine $\widehat{\operatorname{sl}}(2)$ current algebra at level $k=-1 / 2$. In terms of the fields $\beta$ and $\gamma$ the three currents are constructed as follows,

$$
E_{+}^{1}(z)=\frac{1}{2} \beta^{2}(z), \quad H^{1}(z)=-\frac{1}{2}(\beta \gamma)(z), \quad E_{-}^{1}(z)=-\frac{1}{2} \gamma^{2}(z) .
$$

Consequently, we can decompose the generating functions (3.5) into characters of irreducible representations of $\widehat{\mathrm{sl}}(2)_{-1 / 2}$. In case of $\chi^{(0)}$, for example, the decomposition is given by

$$
\chi^{(0)}(q, y)=\frac{\eta(q)}{\theta_{4}\left(q, y^{1 / 2}\right)}=\chi_{0}^{k=-1 / 2}(q, y)+\chi_{1 / 2}^{k=-1 / 2}(q, y) .
$$

The two characters on the right hand side belong to irreducible highest weight representations with lowest weight $h=\epsilon \in\{0,1 / 2\}$,

$$
\chi_{\epsilon}^{k=-1 / 2}(q, y)=\frac{\eta(q)}{2}\left[\frac{1}{\theta_{4}\left(q, y^{1 / 2}\right)}+(-1)^{2 \epsilon} \frac{1}{\theta_{3}\left(q, y^{1 / 2}\right)}\right] .
$$

Let us note that the ground states transform in representations of $\operatorname{spin} j=\epsilon$. Nevertheless, we shall continue to think of the subscript of $\chi$ as the conformal weight rather than the spin. Similar decomposition formulas exist for all the other functions (3.5). All of them are related by the action of spectral flow automorphisms. In particular, we have

$$
\chi^{(1 / 2)}=\chi_{\sigma ;+}^{k=-1 / 2}+\chi_{\sigma ;-}^{k=-1 / 2} \quad \text { with } \chi_{\sigma ; \pm}(q, y)=\frac{y^{1 / 4} \eta(q)}{2}\left[\frac{1}{i \theta_{1}\left(q, y^{-1 / 2}\right)} \pm \frac{1}{\theta_{2}\left(q, y^{-1 / 2}\right)}\right] .
$$

The two characters on the left hand side belong to the two irreducible lowest weight representations of the current algebra with spin $j=1 / 4$ and $j=3 / 4$. Their ground states have the same conformal weight $h=-1 / 8$.

We are now ready to discuss the relevant bulk modular invariant for the theory (1.5) with $S>0$. Let us begin with the product of $S \beta \gamma$-systems and $2 S+2$ real fermions. This theory contains a group $\Gamma_{S}$ of simple currents that consists of all elements $\gamma$ of the form

$$
\gamma=\left[\epsilon_{1}, \ldots \epsilon_{S} ; \epsilon_{S+1}, \ldots, \epsilon_{3 S+2}\right] \quad \text { with } \epsilon_{i} \in\{0,1 / 2\} \text { and } \epsilon \equiv \sum_{i=1}^{3 S+2} \epsilon_{i}=0 \bmod 1 .
$$

The first $S$ entries of $\gamma$ denote sectors of the $\beta \gamma$-system while the remaining ones are representing sectors in the Ising models. Together, the elements $\gamma$ generate the abelian group $\Gamma_{S} \cong \mathbb{Z}_{2}^{3 S+1}$.

Let us first deal with the sector involving representations with vanishing spectral flow, $\nu=0$. Under the action of $\Gamma_{S}$, the sectors with vanishing monodromy charge split into two orbits of maximal length. Hence we are led to the following contribution to the partition function,

$$
Z_{S, 0}^{\mathrm{FF}}\left(q, y_{1}, \ldots, y_{n}\right)=\left|\sum_{\gamma \in \Gamma_{S}} \chi_{\gamma \times[0, \ldots, 0 ; 0, \ldots, 0]}\right|^{2}+\left|\sum_{\gamma \in \Gamma_{S}} \chi_{\gamma \times[0, \ldots, 0 ; 0, \ldots, 0,1 / 2]}\right|^{2} .
$$

However, the total theory has to be invariant under the spectral flow symmetry. Hence we have to add twisted contributions $Z_{S, \nu}^{\mathrm{FF}}$. It was already mentioned above that all the bosonic 
ghosts and all the fermions have to have identical periodicity conditions in order to not to spoil $\operatorname{osp}(2 S+2 \mid 2 S)$ symmetry. Consequently the spectral flow must act diagonally, i.e. simultaneously on all sectors, by half-integer shifts 7 In the fermionic factors, spectral flow by $\nu=1 / 2$ brings us to $\sigma$-representations. Integer units of the spectral flow, however, do not give anything new. In the ghost sectors things works differently because the application of a diagonal spectral flow leads to an infinite number of new representations constructed from the ground states $|\nu\rangle$ for $\nu \in \frac{1}{2} \mathbb{Z}$. Since the orbits of the half-integer spectral flow representations possess a stabilizer subgroup $\mathcal{S}$ of order $|\mathcal{S}|=2^{2 S+1}$ with respect to the action of $\Gamma_{1}$ we finally end up with the partition function

$$
\begin{aligned}
Z_{S}^{\mathrm{FF}}\left(q, y_{1}, \ldots, y_{S}\right)= & \sum_{\nu \in \frac{1}{2} \mathbb{Z}} Z_{S, \nu}^{\mathrm{FF}}\left(q, y_{1}, \ldots, y_{S}\right) \\
=\sum_{\nu \in \mathbb{Z}} & {\left[\left|\sum_{\gamma \in \Gamma_{S}} \chi_{\gamma \times[0, \ldots, 0 ; 0, \ldots, 0]}^{(\nu)}\right|^{2}+\left|\sum_{\gamma \in \Gamma_{S}} \chi_{\gamma \times[0, \ldots, 0 ; 0, \ldots, 0,1 / 2]}^{(\nu)}\right|^{2}\right] } \\
& +2^{2 S+1} \sum_{\nu \in \mathbb{Z}+\frac{1}{2}}\left|\prod_{a=1}^{S} \chi^{(\nu)}\left(q, y_{a}\right)\left(\chi_{\sigma}(q)\right)^{2 S+2}\right|^{2} .
\end{aligned}
$$

Here, the superscript $(\nu)$ on a function $f\left(y_{i}\right)$ of $S$ variables $y_{i}$ is defined through the prescription $f^{(\nu)}\left(y_{i}\right)=q^{-S \nu^{2} / 2} f\left(y_{i} q^{-2 \nu}\right)$.

The rest of our analysis in this section is now carried out for the special case of $S=1$. Generalizations to larger values of $S$ shall be differed to the next section. The state space of our orbifold theory can be equipped with the action of an affine $\widehat{\operatorname{osp}}(4 \mid 2)$ Lie superalgebra. We have already spelled out expressions for the first set of $\mathrm{sl}(2)$ currents in equation (3.6) above. The currents associated with the other two copies if sl(2) take the form

$$
\begin{aligned}
& E_{ \pm}^{2}(z)=\frac{1}{2 i}\left[\left(\psi_{1} \psi_{3}\right)-\left(\psi_{2} \psi_{4}\right) \pm i\left(\left(\psi_{1} \psi_{4}\right)+\left(\psi_{2} \psi_{3}\right)\right)\right] \\
& H^{2}(z)=\frac{1}{2 i}\left(\left(\psi_{3} \psi_{4}\right)+\left(\psi_{1} \psi_{2}\right)\right), \quad H^{3}(z)=\frac{1}{2 i}\left(\left(\psi_{3} \psi_{4}\right)-\left(\psi_{1} \psi_{2}\right)\right), \\
& E_{ \pm}^{3}(z)=\frac{1}{2 i}\left[\left(\psi_{1} \psi_{3}\right)+\left(\psi_{2} \psi_{4}\right) \pm i\left(\left(\psi_{1} \psi_{4}\right)-\left(\psi_{2} \psi_{3}\right)\right)\right] .
\end{aligned}
$$

They generate two commuting copies of the current algebra $\widehat{\mathrm{sl}}(2)_{1}$. In addition, we can introduce the eight fermionic currents through the following expressions

$$
\begin{array}{lll}
F^{+++}(z)=i \beta\left(\psi_{3}+i \psi_{4}\right)(z), & F^{+--}(z)=i \beta\left(\psi_{3}-i \psi_{4}\right)(z), \\
F^{++-}(z)=i \beta\left(\psi_{1}+i \psi_{2}\right)(z), & F^{+-+}(z)=i \beta\left(\psi_{1}-i \psi_{2}\right)(z),
\end{array}
$$

and similarly for $F^{- \pm \pm}(z)$ with the field $\beta$ in the above formulas exchanged with $\gamma$. Note that all terms that contribute to the seventeen currents are quadratic in the basic fields. Since by

\footnotetext{
${ }^{7}$ It is worth mentioning that these diagonal spectral flow transformations are also the only ones which commute with the action of the orbifold group. Note also that half-integer spectral flow on ghosts and fermions implies integer spectral flow on the currents such as those defined in eq. 3.6) and below.
} 
construction these basic fields are either all in the Neveu-Schwarz sector or in the Ramond sector, the currents obey periodic boundary conditions on the entire state space. In order to rewrite the partition function of our bulk theory in terms of affine $\widehat{\mathrm{osp}}(4 \mid 2)$ characters, we recall the following formulas for characters of an $\widehat{\operatorname{sl}}(2)$ currents algebra at level $k=1$,

$$
\chi_{0}^{k=1}(q, z)=\frac{\theta_{3}\left(q^{2}, z\right)}{\eta(q)} \quad, \quad \chi_{1 / 2}^{k=1}(q, z)=\frac{\theta_{2}\left(q^{2}, z\right)}{\eta(q)} .
$$

The lower index $j=0,1 / 2$ now denotes the spin of representations of the $\widehat{\operatorname{sl}}(2)$ current algebra. In terms of characters of the bosonic current algebras, the orbifold partition function reads

$$
\begin{aligned}
Z_{S=1}^{\mathrm{FF}}\left(q, z_{i}\right)= & \sum_{\nu=-\infty}^{\infty}\left|\chi_{(0 ; 0,0)}^{(\nu)}\left(q, z_{i}\right)+\chi_{\left(\frac{1}{2} ; \frac{1}{2}, \frac{1}{2}\right)}^{(\nu)}\left(q, z_{i}\right)\right|^{2}+ \\
& +\sum_{\nu=-\infty}^{\infty}\left|\chi_{\left(0 ; \frac{1}{2}, \frac{1}{2}\right)}^{(\nu)}\left(q, z_{i}\right)+\chi_{\left(\frac{1}{2} ; 0,0\right)}^{(\nu)}\left(q, z_{i}\right)\right|^{2}
\end{aligned}
$$

where the action of the spectral flow involves the first variable $z_{1}=y$ only and we have defined

$$
\chi_{\left(j_{1} ; j_{2}, j_{3}\right)}\left(q, z_{i}\right)=\chi_{j_{1}}^{k=-\frac{1}{2}}\left(q, z_{1}\right) \chi_{j_{2}}^{k=1}\left(q, z_{2}\right) \chi_{j_{3}}^{k=1}\left(q, z_{3}\right) .
$$

To compare the formula (3.12) with our previous expression (3.10) one has to specialize to $z_{2}=z_{3}=1$. Going one step further we can combine characters of the bosonic current algebra into $\widehat{\mathrm{osp}}(4 \mid 2)_{1}$ characters according to,

$$
\begin{aligned}
\chi_{\{0\}}\left(q, z_{i}\right) & =\chi_{(0 ; 0,0)}\left(q, z_{i}\right)+\chi_{\left(\frac{1}{2} ; \frac{1}{2}, \frac{1}{2}\right)}\left(q, z_{i}\right), \\
\chi_{\{1 / 2\}}\left(q, z_{i}\right) & =\chi_{\left(0 ; \frac{1}{2}, \frac{1}{2}\right)}\left(q, z_{i}\right)+\chi_{\left(\frac{1}{2} ; 0,0\right)}\left(q, z_{i}\right) .
\end{aligned}
$$

The results of this section may then be summarized through the following simple formula

$$
Z_{S=1}^{\mathrm{FF}}\left(q, z_{i}\right)=\sum_{\nu=-\infty}^{\infty}\left|\chi_{\{0\}}^{(\nu)}\left(q, z_{i}\right)\right|^{2}+\sum_{\nu=-\infty}^{\infty}\left|\chi_{\{1 / 2\}}^{(\nu)}\left(q, z_{i}\right)\right|^{2}
$$

i.e. the orbifold partition function is the charge conjugate modular invariant partition function for the sectors $\{0\}$ and $\{1 / 2\}$ of the $\widehat{\mathrm{osp}}(4 \mid 2)_{1}$ current algebra. It is remarkable that spectral flow relates all the representations occurring here and that the fusion is purely abelian [28. In contrast to other WZNW theories on supergroups [29, 30, 31, 32 this guarantees the existence of an "irreducible" theory without logarithmic correlation functions. By fermionizing the $\beta \gamma$ systems and keeping additional zero-modes, however, one can as well construct a "logarithmic lift" of the theory [33] (see also [30]).

\subsection{Boundary conditions and their spectra}

In the next step we wish to discuss boundary conditions in the orbifold theory constructed above. We will focus on a particular brane. Our choice might seem a bit ad hoc at first, 
but will later turn out to be deformed into the space-filling brane of the PCM. As before, we treat the cases $S=0$ and $S=1$ in some detail and postpone comments on higher values of $S$ to the following section.

In the case $S=0$ we need to construct a brane in the orbifold (3.2) which corresponds to a Neumann brane in the free boson theory at large radius. But in this case the deformation is well known. When we reduce the radius from $R=\infty$ to $R=1$ we pass the self-dual radius where Neumann and Dirichlet branes cannot be distinguished and get exchanged by T-duality. Consequently the brane we would like to describe in the free boson theory at $R=1$ is the Dirichlet brane which has the spectrum

$$
Z_{D}^{R=1}(q)=\sum_{w \in \mathbb{Z}} \frac{q^{\frac{w^{2}}{2}}}{\eta(q)}=\frac{\theta_{3}(q)}{\eta(q)} .
$$

We will now show how the same spectrum can be obtained from the orbifold model.

The Ising model is the simplest of the Virasoro minimal models. It has precisely three different conformal boundary conditions, one for each of irreducible representations $[0],[1 / 2]$ and $[\sigma]=[1 / 16]$. Here and in the following we shall labels boundary conditions and sectors by the same symbol. The spectrum of excitations between any two of these boundary conditions is described by the respective fusion rules [34]. In order to make contact with the bosonic description, let us try to rewrite the partition function (3.16) through characters (3.1) of the two Ising models. After simple manipulations we find

$$
Z_{D}^{R=1}(q)=\frac{\theta_{3}(q)}{\eta(q)}=\chi_{(0,0)}+\chi_{(1 / 2,1 / 2)}+\chi_{(0,1 / 2)}+\chi_{(1 / 2,0)} .
$$

The spectrum we find can be considered as the orbit of the sum $[0,0] \oplus[0,1 / 2]$ under the action of the orbifold group $\Gamma_{0}$. Since $[0,0] \oplus[0,1 / 2]$ is precisely the fusion product $[\sigma, 0] \times[\sigma, 0]$ we conclude that the desired point-like brane at $R=1$ descends under the orbifold construction from the boundary condition $[\sigma, 0]$ in the product of two Ising models. The conclusion is fully consistent with the free fermion construction of the bosonic current $J \sim \psi_{1} \psi_{2}$ of the $R=1$ model. In fact, as is well known, the boundary label $[0, \sigma]$ corresponds to the gluing conditions

$$
\psi_{1}(z)=-\bar{\psi}_{1}(\bar{z}) \quad \psi_{2}(z)=\bar{\psi}_{2}(\bar{z}) \quad(\text { for } z=\bar{z})
$$

in the underlying free fermion description. The sign in the gluing condition for the first fermionic field is associated with the non-trivial boundary label $[\sigma]$. It implies that the current $J \sim \psi_{1} \psi_{2}$ satisfies Dirichlet boundary conditions $J=-\bar{J}$ all along the boundary.

Let us now turn our attention to the case $S=1$. We would like to focus on a brane which is associated with the twisted gluing conditions

$$
J^{1}(z)=\bar{J}^{1}(\bar{z}), \quad J^{2}(z)=\bar{J}^{3}(\bar{z}), J^{3}(z)=\bar{J}^{2}(\bar{z})
$$

for the bosonic currents $J^{i}=E_{a}^{i} t^{a}$ all along the boundary at $z=\bar{z}$. The underlying gluing automorphism $\Omega$ permutes the second and third copy of $\operatorname{sl}(2)$ in the bosonic subalgebra. It 
can easily be seen that $\Omega$ extends to an involution on the entire superalgebra osp(4|2). The corresponding gluing conditions for fermionic currents read,

$$
F^{\xi \pm \pm}(z)=\bar{F}^{\xi \pm \pm}(\bar{z}) \quad F^{\xi \pm \mp}(z)=\bar{F}^{\xi \mp \pm}(\bar{z}) .
$$

A quick look back at the free field realization of the currents (3.10) suggests to implement the boundary conditions (3.19) and (3.20) through the following gluing prescription for the fundamental field multiplet,

$$
\psi_{1}(z)=-\bar{\psi}_{1}(\bar{z}), \quad \psi_{i}(z)=\bar{\psi}_{i}(\bar{z}) \quad(i \neq 1), \quad \beta_{a}(z)=\bar{\beta}_{a}(\bar{z}), \quad \gamma_{a}(z)=\bar{\gamma}_{a}(\bar{z}) .
$$

Indeed, equations (3.21) reproduce the permutation of currents displayed in eqs. (3.19) and (3.20) upon insertion into eqs. (3.10).

Just as in the case $S=0$ above, having a non-trivial gluing condition for the fermion is associated with the occurrence of the brane label $\sigma$ in the Ising model description. Hence we propose that the desired orbifold brane may be constructed from the brane $B=[0,0 ; \sigma, 0,0,0]$ in the covering theory. The spectrum for the latter is again given by fusion, and taking the orbit with respect to the orbifold group $\Gamma_{1}$ one easily arrives at

$$
Z_{B ; S=1}^{\mathrm{FF}}=\sum_{\gamma \in \Gamma_{1}}\left[\chi_{\gamma \times[0,0 ; 0,0,0,0]}+\chi_{\gamma \times[0,0 ; 0,1 / 2,0,0]}\right] .
$$

For later convenience this result may also be rewritten in terms of irreducible characters of the underlying bosonic current algebra, leading to

$$
Z_{B ; S=1}^{\mathrm{FF}}\left(q, z_{i}\right)=\chi_{(0 ; 0,0)}+\chi_{\left(0 ; \frac{1}{2}, \frac{1}{2}\right)}+\chi_{\left(\frac{1}{2} ; \frac{1}{2}, \frac{1}{2}\right)}+\chi_{\left(\frac{1}{2} ; 0,0\right)}=\chi_{\{0\}}+\chi_{\{1 / 2\}} .
$$

In the second step we have combined characters of the bosonic subalgebra into characters of the full $\widehat{\mathrm{osp}}(4 \mid 2)_{1}$, using the formulas (3.13) and (3.14). The spectrum of the orbifold brane preserves the affine Lie superalgebra, as desired. We also note that our partition function $Z_{B ; S=1}^{\mathrm{FF}}(q)$ is identical to the one that appeared in the work of Candu and Saleur [23, 24]. We shall now see that it is related through a deformation to the partition function of the volume filling brane in the PCM model.

\subsection{Casimir decomposition in the free GN model}

Having found the full spectrum of an osp $(4 \mid 2)$ symmetric brane in the free field theory (1.5), our next task is to expand it in terms of the characters $\chi_{\lambda}^{K}$. In other words, we need to find the branching functions $\psi_{\Lambda}^{K}(q)$ in the decomposition,

$$
\tilde{Z}=Z_{B ; S=1}^{\mathrm{FF}}\left(q, z_{i}\right)=\sum_{\Lambda} \chi_{\Lambda}^{K}\left(z_{1}, z_{2}, z_{3}\right) \tilde{\psi}_{\Lambda}^{K}(q) .
$$

This expansion is of the same form (2.17) as in the PCM at $R=\infty$. Only the branching functions $\tilde{\psi}^{K}$ are different. The following short analysis will show that they read

$$
\begin{aligned}
\tilde{\psi}_{\left[j_{1}, j_{2}, j_{3}\right]}^{K}(q)= & \frac{1}{\eta(q) \phi^{3}(q)} \sum_{n, m=0}^{\infty}(-1)^{n+m} q^{\frac{m}{2}\left(m+4 j_{1}+2 n+1\right)+j_{1}+\frac{n}{2}} \\
& \times\left(q^{\left(j_{2}-\frac{n}{2}\right)^{2}}-q^{\left(j_{2}+\frac{n}{2}+1\right)^{2}}\right)\left(q^{\left(j_{3}-\frac{n}{2}\right)^{2}}-q^{\left(j_{3}+\frac{n}{2}+1\right)^{2}}\right) .
\end{aligned}
$$


Before we derive this formula, we wish to comment on its implications. A short look back to formula (2.18) reveals a remarkable similarity between the two branching functions of the partition functions $Z$ of the PCM at $R=\infty$ and $\tilde{Z}$ of the free fields theory (1.5). In fact, they are identical up to an overall prefactor,

$$
\psi_{\left[j_{1}, j_{2}, j_{3}\right]}^{K}(q)=q^{2 j_{1}\left(j_{1}-1\right)-j_{2}\left(j_{2}+1\right)-j_{3}\left(j_{3}+1\right)} \tilde{\psi}_{\left[j_{1}, j_{2}, j_{3}\right]}^{K}(q)
$$

For the time being this equation may simply be considered a curious observation regarding the similarities of the two Casimir decompositions. We shall explain in the next subsection how it relates to the claim that the boundary spectrum for the PCM at $R=\infty$ may be obtained by the current-current perturbation (1.6) from the free field theory (1.5).

In order to calculate the branching functions $\tilde{\psi}^{K}$ from the partition function $\tilde{Z}$, we proceed as in section 2.3. In a first step we shall expand $\tilde{Z}$ in terms of characters of the bosonic subalgebra osp $(4 \mid 2)_{\overline{0}}$. Then we combine the bosonic building blocks into characters of Kac modules for $\operatorname{osp}(4 \mid 2)$. The resulting expression for the branching function will require only very little additional analysis in order to cast them into the form (3.25).

The decomposition of $\tilde{Z}$ into bosonic characters departs from the representation (3.23) of $\tilde{Z}$ and then employs the following expansion formulas for $\widehat{\mathrm{sl}}(2)$ characters into sums of characters of $\operatorname{sl}(2)$,

$$
\begin{aligned}
\chi_{a}^{k=-\frac{1}{2}}(\tau, u) & =\frac{q^{\frac{1}{24}}}{\phi(q)^{2}} \sum_{k \in \mathbb{N}+a} \chi_{k}(z) \sum_{m=0}^{\infty}(-1)^{m} q^{\frac{m}{2}(m+4 k+1)+k}\left(1-q^{2 m+1}\right) \\
\chi_{a}^{k=1}(\tau, u) & =\frac{1}{\eta(q)} \sum_{m \in \mathbb{N}+a} \chi_{m}(z)\left(q^{m^{2}}-q^{(m+1)^{2}}\right)
\end{aligned}
$$

where $a \in\left\{0, \frac{1}{2}\right\}$. ¿From the equality (3.23) and the two decomposition formulas (3.27) and (3.28) it is clear that $\tilde{Z}$ can be written as

$$
\tilde{Z}=\sum_{\substack{\left(j_{1}, j_{2}, j_{3}\right) \in \frac{1}{2} \mathbb{N}^{3} \\ j_{2}+j_{3} \in \mathbb{N}}} \chi_{\left(j_{1}, j_{2}, j_{3}\right)}\left(z_{1}, z_{2}, z_{3}\right) \tilde{\psi}_{\left(j_{1}, j_{2}, j_{3}\right)}^{B}(q)
$$

where $\chi_{\left(j_{1}, j_{2}, j_{3}\right)}$ are the characters of the irreducible representations of $\operatorname{osp}(4 \mid 2)_{\overline{0}}$, as before, and the branching functions $\tilde{\psi}^{B}$ are given by

$$
\begin{aligned}
\tilde{\psi}_{\left(j_{1}, j_{2}, j_{3}\right)}^{B}(q)=\frac{1}{\eta(q) \phi^{3}(q)} \sum_{m=0}^{\infty}(-1)^{m} q^{\frac{m}{2}\left(m+4 j_{1}+1\right)+j_{1}}\left(1-q^{2 m+1}\right) \\
\times\left(q^{j_{2}^{2}}-q^{\left(j_{2}+1\right)^{2}}\right)\left(q^{j_{3}^{2}}-q^{\left(j_{3}+1\right)^{2}}\right) .
\end{aligned}
$$

Before we proceed let us note that the branching functions $\tilde{\psi}_{\Lambda}^{B}$ possess the following important symmetry properties necessary for a proof in Appendix C,

$$
\tilde{\psi}_{\left(j_{1}, j_{2}, j_{3}\right)}^{B}(q)=-\tilde{\psi}_{\left(-j_{1}-1, j_{2}, j_{3}\right)}^{B}(q)=-\tilde{\psi}_{\left(j_{1},-j_{2}-1, j_{3}\right)}^{B}(q)=-\tilde{\psi}_{\left(j_{1}, j_{2},-j_{3}-1\right)}^{B}(q) .
$$


These imply in particular that $\psi_{\left(j_{1}, j_{2}, j_{3}\right)}^{B}(q)$ vanishes identically if any of the spin labels $j_{a}$ is equal to $j_{a}=-1 / 2$. As in our analysis of the PCM's partition function $Z$ in section 2.3 , we can express all characters of representations of the bosonic subalgebra as infinite linear combinations of the characters of Kac modules. The required formulas can be found in Appendix [C] With their help we now arrive at the following result for $\tilde{\psi}_{\Lambda}^{K}$,

$$
\begin{aligned}
\tilde{\psi}_{\left[j_{1}, j_{2}, j_{3}\right]}^{K}(q)= & \frac{1}{\eta(q) \phi^{3}(q)} \sum_{n, m=0}^{\infty}(-1)^{n+m} q^{\frac{m}{2}\left(m+4 j_{1}+1\right)+j_{1}+m n+\frac{n}{2}}\left(1-q^{2 m+1}\right) \\
& \times \sum_{k=0}^{\left[\frac{n}{2}\right]}\left(q^{\left(j_{2}-\frac{n}{2}+k\right)^{2}}-q^{\left(j_{2}+\frac{n}{2}-k+1\right)^{2}}\right)\left(q^{\left(j_{3}-\frac{n}{2}+k\right)^{2}}-q^{\left(j_{3}+\frac{n}{2}-k+1\right)^{2}}\right) \\
= & \frac{1}{\eta(q) \phi^{3}(q)} \sum_{n, m=0}^{\infty}(-1)^{n+m} q^{\frac{m}{2}\left(m+4 j_{1}+2 n+1\right)+j_{1}+\frac{n}{2}}\left(1-q^{2 m+1}\right) \\
& \times\left(q^{\left(j_{2}-\frac{n}{2}\right)^{2}}-q^{\left(j_{2}+\frac{n}{2}+1\right)^{2}}\right)\left(q^{\left(j_{3}-\frac{n}{2}\right)^{2}}-q^{\left(j_{3}+\frac{n}{2}+1\right)^{2}}\right) \sum_{k=0}^{\infty} q^{(2 m+1) k} .
\end{aligned}
$$

The sum over $k$ at the end of this formula is a simple geometric series which cancels the last term in the first line. Thereby, we recover the expression (3.25) we spelled out at the beginning of this subsection.

\subsection{Deformation from free GN model to free PCM}

The main result of our analysis so far was summarized concisely in eq. (3.26). In order to fully appreciate its content, let us review a few results from [22]. In that paper, the deformation of conformal weights was studied for the WZNW model on PSL $(2 \mid 2)$. Many of the central results of 22], however, hold much more generally for models whose symmetries are described by an affine Lie superalgebra with vanishing dual Coxeter number.

To begin with, let us specify the bulk perturbation we would like to consider. As we shall argue momentarily, it is generated by the field,

$$
\Phi=\sum \kappa_{\mu \nu} J^{\mu}(z) \Omega\left(\bar{J}^{\nu}(\bar{z})\right)
$$

where the summation extends over all 17 bosonic and fermionic directions. The automorphism $\Omega$ we inserted here is the same as the gluing automorphism that was defined implicitly through our gluing conditions (3.19) and (3.20) in section 3.2. Note that the perturbing operator $\Phi$ breaks the global symmetry from osp $(4 \mid 2) \otimes \operatorname{osp}(4 \mid 2)$ of the free GN model (1.5) to the twisted diagonal subalgebra. In other words, the symmetry transformations of the perturbed model are generated by elements of the form $X \otimes 1+1 \otimes \Omega(X)$. This means that any perturbing operator of the form $\Phi$ preserves half of the global bulk symmetries. What depends on the choice of the automorphism $\Omega$ is the precise set of transformations that is preserved. Similar statements can be made about boundary conditions. As we discussed in section 3.2, the boundary theory we put forward to compare with the boundary spectrum 
of the PCM required to select a non-trivial gluing automorphism $\Omega$. If this gluing automorphism would differ from the automorphism $\Omega$ in the definition of $\Phi$, then the boundary condition and the deformation would preserve different sets of symmetry generators. Hence, the deformed boundary theory would no longer possess a global osp(4|2) symmetry. Such a theory could be conformal, but it cannot be equivalent to the boundary PCM. Therefore, we know that the perturbing operator $\Phi$ must involve the same automorphism $\Omega$ that appeared in the gluing condition for currents at the boundary. An explicit formula for the operator $\Phi$ in terms of free fields is derived at the end of appendix D. The resulting expression agrees with the formula for $\mathcal{S}^{\text {int }}$ we anticipated in the introduction.

Having specified the deforming operator, we are now ready to discuss the properties of the deformation it generates. Here we shall closely follow the the recent analysis in 22 . Everything we shall claim below is based on a rather simple mathematical result that was first formulated and exploited in the work of Bershadsky et. al. [16] for psl $(\mathrm{N} \mid \mathrm{N})$, but holds equally for $\operatorname{osp}(2 S+2 \mid 2 S)$. Consider some osp $(2 S+2 \mid 2 S)$ invariant $\Delta$, such as e.g. a conformal weight, and suppose that $\Delta$ may be written as $\Delta=C_{a b c} f^{a b c}$ where $f^{a b c}$ are the structure constants of $\operatorname{osp}(2 S+2 \mid 2 S)$ and $C_{a b c}$ are some numbers. Then $\Delta$ can be shown to vanish.

We would like to apply this mathematical lemma to the computation of conformal weights. To evaluate the change of conformal weights away from the free GN model, we perform a perturbative analysis of 2-point functions in our theory. In any such computation of perturbed correlators, the initial step is to remove all the current insertions through current algebra Ward identities. In the process, pairs of currents get contracted using

$$
J^{\mu}(z) J^{\nu}(w)=\frac{i f_{\sigma \nu}^{\mu \nu}}{z-w} J^{\sigma}(w)+\frac{k \kappa^{\mu \nu}}{(z-w)^{2}}+\ldots \sim \frac{k \kappa^{\mu \nu}}{(z-w)^{2}} .
$$

The first equality is the usual operator product for $\operatorname{osp}(4 \mid 2)$ currents. Since we are only interested in computing the invariants $h$, we can drop all terms that involve the structure constants $f$ of the Lie superalgebra osp $(4 \mid 2)$. This applies to the first term in the above operator product which distinguishes the non-abelian currents from the abelian algebra of flat target spaces. Here and in the following we shall use the symbol $\sim$ to mark equalities that are true up to terms involving structure constants. In conclusion, we have seen that, as far as the computation of conformal dimensions is concerned, we may neglect the non-abelian nature of the currents $J^{\mu}$. Obviously, this leads to drastic simplifications of the perturbative expansion.

In 22] several other statements were needed to study a deformation that preserved simultaneously both left and right global symmetries. The perturbation (1.6) we consider here, however, is of a much simpler type. We can therefore directly move on to evaluate the conformal dimension of boundary fields. Unlike in 22, the following arguments apply to all boundary conditions, as long as they preserve the affine $\widehat{\mathrm{osp}}(4 \mid 2)$ symmetry. It does not require any further assumptions on the localization of the brane. Let $\Psi$ be some multiplet of boundary fields transforming in a representation $\Lambda$ of $\operatorname{osp}(4 \mid 2)$. We denote by $h_{0}(\Psi)$ the conformal weight of $\Psi$ at the WZ-point. Upon deformation with the field (3.32), the weight 
of $\Psi$ behaves as

$$
h(\Psi)=h_{0}(\Psi)-\frac{1}{2} \frac{g^{2}}{1+g^{2}} C_{2}(\Lambda)=h_{0}(\Psi)+\frac{1}{2}\left(\frac{1}{R^{2}}-1\right) C_{2}(\Lambda)
$$

where $C_{2}$ is the quadratic Casimir element of the Lie superalgebra osp $(4 \mid 2)$, as before.

Through the Casimir decomposition (3.24) of the boundary partition function $\tilde{Z}$ we have separated all boundary fields according to their osp $(4 \mid 2)$ transformation law. This now allows us to evaluate the shift of conformal weights for entire blocks rather than individual field multiplets. More concretely, the conformal weights of all fields that are counted by the branching function $\tilde{\psi}_{\left[j_{1}, j_{2}, j_{3}\right]}^{K}$ undergo the same shift by 8

$$
\delta_{g}(h)=-\frac{1}{2} \frac{g^{2}}{1+g^{2}} C_{2}\left[j_{1}, j_{2}, j_{3}\right]=\frac{g^{2}}{1+g^{2}}\left(2 j_{1}\left(j_{1}-1\right)-j_{2}\left(j_{2}+1\right)-j_{3}\left(j_{3}+1\right)\right)
$$

upon perturbation with $\Phi$. Thereby, we can spell out the boundary spectrum of the perturbed model for any choice of $g^{2}=R^{2}-1$,

$$
\begin{aligned}
\tilde{Z}_{R}\left(q, z_{i}\right)= & q^{-\frac{1}{24}} \sum_{j_{i}} \chi_{\left[j_{1}, j_{2}, j_{3}\right]}^{K}\left(z_{1}, z_{2}, z_{3}\right) \times \\
& \times q^{\left(1-\frac{1}{R^{2}}\right)\left(2 j_{1}\left(j_{1}-1\right)-j_{2}\left(j_{2}+1\right)-j_{3}\left(j_{3}+1\right)\right)} \tilde{\psi}_{\left[j_{1}, j_{2}, j_{3}\right]}^{K}(q) .
\end{aligned}
$$

For irrational values of the parameter $R$, the boundary spectrum is rather rich, containing irrational conformal weights. But as we reach the special value $R=\infty$, all conformal weights become integers. Equation (3.26) tells us even more: At this particular point, the perturbed boundary partition function coincides with the partition function $Z$ of volume filling branes in the PCM on the supersphere $S^{3 \mid 2}$ in the limit $R \rightarrow \infty$. For a few selected multiplets, the deformation from $R=\infty$ to $R=1$ had been carried out in [24. By performing the Casimir decompositions explicitly, we were able to extend such studies to the entire spectrum.

\section{Generalization for higher-dimensional superspheres}

The aim of this section is to outline how the previous analysis may be extended to higher dimensional superspheres. We shall provide explicit formulas for the relevant boundary spectra of the PCM at $R=\infty$ and for the free field theory (1.5). The latter are expressed in terms of characters of the affine $\widehat{\mathrm{osp}}(2 S+2 \mid 2 S)$ superalgebra at $k=1$. Note that the level does not depend on $S$. Since we have not attempted to construct the branching functions $\psi_{\Lambda}$ and $\tilde{\psi}_{\Lambda}$ for the decomposition with respect to the global osp $(2 S+2 \mid 2 S)$ symmetry, we shall content ourselves with a few non-trivial tests. These are discussed in the second subsection. We believe that a full analysis, as in the case of $S=1$, is possible but cumbersome.

\footnotetext{
${ }^{8}$ Let us recall that all irreducible multiplets that can be tied together in an indecomposable representation must have identical Casimir eigenvalues, see appendix A.
} 


\subsection{Partition functions for superspheres at $R=1, \infty$}

The first task is to spell out the spectrum of the PCM with Neumann boundary conditions at $R=\infty$. It turns out that our formula (2.16) for $S=1$ admits the following straightforward generalization,

$$
Z_{N ; S}^{\mathrm{PCM}}=q^{-\frac{1}{24}} Z_{0}^{(S)} \phi(q) \prod_{n=1}^{\infty} \frac{\prod_{m=1}^{S}\left(1+y_{m} q^{n}\right)\left(1+y_{m}^{-1} q^{n}\right)}{\prod_{k=1}^{S+1}\left(1-x_{k} q^{n}\right)\left(1-x_{k}^{-1} q^{n}\right)} .
$$

Here, the subscript $N$ stands for Neumann boundary conditions and the minisuperspace contribution is given by

$$
Z_{0}^{(S)}=\lim _{t \rightarrow 1}\left(1-t^{2}\right) \frac{\prod_{m=1}^{S}\left(1+y_{m} t\right)\left(1+y_{m}^{-1} t\right)}{\prod_{k=1}^{S+1}\left(1-x_{k} t\right)\left(1-x_{k}^{-1} t\right)} .
$$

As before, the factor $Z_{0}^{(S)}$ describes the space of functions on $S^{2 S+1 \mid 2 S}$. As mentioned above, we have not performed the analysis of section 2.3 for the more general partition function $Z_{N ; S}^{\mathrm{PCM}}$, though this would surely be possible.

Next let us turn to the free GN model (1.5). Large parts of our analysis of the bulk spectrum were already performed for generic $S$. Once more, the theory possesses an affine $\widehat{\mathrm{osp}}(2 S+2 \mid 2 S)$ symmetry with level $k=1$ (see appendix D for an explicit construction of the generators in terms of the basic fields). The bulk theory can be shown to possess a symmetry preserving boundary condition whose spectrum closely resembles eq. (3.23). Before we are able to spell out the details, we shall quote from [35] the following expressions for characters of the affine Lie algebra $\widehat{\operatorname{so}}(2 S+2)$ at level $k=1$,

$$
\begin{aligned}
& \chi_{(0)}^{\text {so }}\left(q, x_{i}\right)=\frac{1}{2 \eta(q)^{S+1}}\left(\prod_{i=1}^{S+1} \theta_{3}\left(q, x_{i}\right)+\prod_{i=1}^{S+1} \theta_{4}\left(q, x_{i}\right)\right), \\
& \chi_{(\mathrm{f})}^{\text {so }}\left(q, x_{i}\right)=\frac{1}{2 \eta(q)^{S+1}}\left(\prod_{i=1}^{S+1} \theta_{3}\left(q, x_{i}\right)-\prod_{i=1}^{S+1} \theta_{4}\left(q, x_{i}\right)\right) .
\end{aligned}
$$

Note that $\widehat{\mathrm{so}}(2 S+2)_{1}$ is part of the bosonic subalgebra of $\widehat{\mathrm{osp}}(2 S+2 \mid 2 S)_{1}$. Similarly, we also need the corresponding characters of the affine $\widehat{\mathrm{sp}}(2 S)$ at $k=-\frac{1}{2}$

$$
\begin{aligned}
& \chi_{(0)}^{\mathrm{sp}}\left(q, y_{i}\right)=\frac{\eta(q)^{S}}{2}\left(\frac{1}{\prod_{i=1}^{S} \theta_{4}\left(q, y_{i}\right)}+\frac{1}{\prod_{i=1}^{S} \theta_{3}\left(q, y_{i}\right)}\right) \\
& \chi_{(\mathrm{f})}^{\mathrm{sp}}\left(q, y_{i}\right)=\frac{\eta(q)^{S}}{2}\left(\frac{1}{\prod_{i=1}^{S} \theta_{4}\left(q, y_{i}\right)}-\frac{1}{\prod_{i=1}^{S} \theta_{3}\left(q, y_{i}\right)}\right) .
\end{aligned}
$$

The characters we have just listed, furnish the basic building blocks for the relevant characters of our superalgebra $\widehat{\operatorname{osp}}(2 S+2 \mid 2 S)_{1}$ at level $k=1$,

$$
\begin{aligned}
& \chi_{\{0\}}^{\mathrm{osp}}=\chi_{(0)}^{\mathrm{so}} \chi_{(0)}^{\mathrm{sp}}+\chi_{(\mathrm{f})}^{\mathrm{so}} \chi_{(\mathrm{f})}^{\mathrm{sp}}, \\
& \chi_{\{\mathrm{ff}\}}^{\mathrm{osp}}=\chi_{(\mathrm{f})}^{\mathrm{so}} \chi_{(0)}^{\mathrm{sp}}+\chi_{(0)}^{\mathrm{so}} \chi_{(\mathrm{f})}^{\mathrm{sp}} .
\end{aligned}
$$


For a particular choice of boundary conditions in the free field theory (1.5) the boundary partition function takes the following form

$$
Z_{B ; S}^{\mathrm{FF}}\left(q, z_{i}\right)=\chi_{\{0\}}^{\mathrm{osp}}+\chi_{\{\mathrm{f}\}}^{\mathrm{osp}}=\frac{1}{\eta(q)} \frac{\prod_{i=1}^{S+1} \theta_{3}\left(q, x_{i}\right)}{\prod_{j=1}^{S} \theta_{4}\left(q, y_{j}\right)},
$$

where the first $S$ variables $z_{i}=y_{i}$ are associated with the symplectic part while the remaining $S+1$ variables $z_{S+i}=x_{i}$ are affiliated with Cartan elements of the orthogonal subalgebra. Eq. (4.6) generalizes equation (3.23) to $S \geq 1$.

\subsection{Test of the duality}

As in the previous section, we would like to show that the two partition functions (4.1) and (4.6) are related to each other by deformation with the interaction term (1.6) or, equivalently, by deforming the radius $R$ of the PCM from $R=\infty$ all the way down to $R=1$. In principle, this may be achieved by repeating our analysis in sections 2.3 and 3.3 above. The first step is to decompose the partition function (4.6) of the PCM at $R=\infty$ in terms of character functions for the global osp $(2 S+2 \mid 2 S)$ symmetry,

$$
Z_{N, S}^{\mathrm{PCM}}=\sum_{\Lambda \in \mathcal{J}} \chi_{\Lambda}^{\mathrm{osp}(2 S+2 \mid 2 S)}\left(z_{i}\right) \psi_{\Lambda}^{(S)}(q)
$$

where $\mathcal{J}$ is the set of all integral dominant labels of $\operatorname{osp}(2 S+2 \mid 2 S)$ that are compatible with the consistency conditions of [36]. The existence of such a decomposition is guaranteed, but in case of $S>1$ explicit formulas for the branching functions $\psi$ would still need to be worked out.

The second step is to pass from $R=\infty$ to finite values of the radius. Since all the general results we outlined in section 3.4 hold for any value of $S$, the boundary partition function of the PCM at radius $R$ reads

$$
Z(R)=\sum_{\Lambda \in \mathcal{J}} \chi_{\Lambda}^{\mathrm{osp}(2 S+2 \mid 2 S)}\left(x_{i}, y_{j}\right) \psi_{\Lambda}^{(S)}(q) q^{\frac{1}{2} \frac{1}{R^{2}} C(\Lambda)}
$$

Here we expressed the partition function through the branching functions $\psi$ at $R=\infty$ rather than through the ones at $R=1$, as in section 3.4. Therefore, the coefficient of the Casimir

element had to be properly adjusted. Note also that we normalized the quadratic Casimir operator such that $C_{2}(\mathrm{f})=1$ for all values of $S$.

For the PCMs on odd dimensional superspheres $S^{2 S+1 \mid 2 S}$ to be dual to the GN model, we would have to find

$$
Z(R=1)=Z_{B ; S}^{\mathrm{FF}}
$$

provided we have correctly identified the appropriate boundary condition in the free field theory (1.5). Throughout the last sections, we have checked relation (4.9) explicitly for $S=1$. It is quite amusing to verify it also in the much simpler case of $S=0$. When $S=0$, 
the decomposition of the partition function at $R=\infty$ into characters of $\operatorname{osp}(2 \mid 0) \cong \operatorname{so}(2)$, takes a particularly simple form,

$$
\begin{aligned}
Z_{N, S=0}^{\mathrm{PCM}} & =q^{-\frac{1}{24}} \phi(q) \sum_{n \in \mathbb{Z}} z^{n} \sum_{k \in \mathbb{Z}} \frac{z^{k}}{\phi(q)^{2}} \sum_{m=0}^{\infty}(-1)^{m}\left(q^{\frac{m+1}{2}(m+2|k|)}-q^{\frac{m+1}{2}(m+2(|k|+1))}\right) \\
& =\frac{1}{\eta(q)} \sum_{n \in \mathbb{Z}} z^{n}=\sum_{n \in \mathbb{Z}} \chi_{n}(z) \psi_{n}^{(0)}(q)
\end{aligned}
$$

with $\chi_{n}(z)=z^{n}$ and $\psi_{n}^{(0)}(q)=1 / \eta(q)$. Following our equation (4.8), the partition function for radius $R$ becomes

$$
Z(R)=\frac{1}{\eta(q)} \sum_{n \in \mathbb{Z}} z^{n} q^{\frac{1}{2} \frac{1}{R^{2}} n^{2}} .
$$

Therefore, at $R=1$ we obtain

$$
Z(R=1)=\frac{1}{\eta(q)} \sum_{n \in \mathbb{Z}} z^{n} q^{\frac{n^{2}}{2}}=\frac{1}{\eta(q)} \sum_{n \in \mathbb{Z}} z^{n} q^{\frac{n^{2}}{2}}=Z_{B ; S=0}^{\mathrm{FF}}(q, z),
$$

in agreement with our general prediction (4.9).

Although we have not been able to find a conclusive proof of (4.9) for $S \geq 2$, we wish to give some additional supporting evidence. To this end, we need a few more details about representations of $\operatorname{osp}(2 S+2 \mid 2 S)$ and the corresponding values of the quadratic Casimir element. The representations we are interested in are labeled by integral dominant highest weights $\Lambda$ of the form

$$
\begin{aligned}
\Lambda= & a_{1} \delta_{1}+a_{2}\left(\delta_{1}+\delta_{2}\right)+\cdots+a_{S}\left(\delta_{1}+\cdots \delta_{S}\right)+a_{S+1} \epsilon_{1}+\cdots+a_{2 S-1}\left(\epsilon_{1}+\cdots \epsilon_{S-1}\right) \\
& +a_{2 S} \frac{\epsilon_{1}+\cdots+\epsilon_{S}-\epsilon_{S+1}}{2}+a_{2 S+1} \frac{\epsilon_{1}+\cdots+\epsilon_{S}+\epsilon_{S+1}}{2}
\end{aligned}
$$

where $\delta_{i}$ and $\epsilon_{j}$ appear in the construction of the weight system of $\operatorname{osp}(2 S+2 \mid 2 S)$ and obey $\left(\epsilon_{i}, \epsilon_{j}\right)=-\left(\delta_{i}, \delta_{j}\right)=\delta_{i j}$. The numerical coefficients $a_{i} \in \mathbb{N}$ must moreover obey some additional consistency conditions that can be found in [36. The value of the quadratic Casimir in the representation of weight $\Lambda$ can now be expressed in terms of the coefficients $a_{i}$ as,

$$
\begin{aligned}
C_{\Lambda}= & (\Lambda, \Lambda+2 \rho)=-\sum_{i=1}^{S}\left(\sum_{j=i}^{S} a_{j}-2 i\right) \sum_{k=i}^{S} a_{k}+\frac{\left(a_{2 S}-a_{2 S+1}\right)^{2}}{4} \\
& +\sum_{i=1}^{S}\left(\sum_{j=i}^{S-1} a_{S+j}+\frac{a_{2 S}+a_{2 S+1}}{2}+2(S+1-i)\right)\left(\sum_{k=i}^{S-1} a_{S+k}+\frac{a_{2 S}+a_{2 S+1}}{2}\right) .
\end{aligned}
$$

The fundamental representation corresponds to $a_{1}=1$ and $a_{i}=0$ for $i \neq 1$ so that $C_{\delta_{1}}=$ $-(1-2)=1$ for all $S$. The value of the quadratic Casimir does not only determine the deformation of conformal weights, see eq. (4.9). It is also needed to compute the conformal weight

$$
h_{\Lambda}=\frac{C_{\Lambda}}{2 k}
$$


of fields that are primary with respect to the underlying affine superalgebra at level $k$. In our case, the level $k$ must be set to $k=1$, as before.

After this preparation we can begin to test equation (4.9). Let us first try to recover the ground states of the free field theory at $R=1$. It is clear that the vacuum state at $R=1$ is obtained by deforming the unique $\operatorname{osp}(2 S+2 \mid 2 S)$ invariant field with weight $h=0$ at $R=\infty$. So, we can turn to the ground states in the second sector of eq. (4.6) right away. From (4.7) we infer that the boundary PCM contains a single field multiplet that transforms in the fundamental representation with $\Lambda=\delta_{1}$ and has conformal weight $h=0$. Under the proposed deformation, the conformal weight of this multiplet is lifted from $h=0$ to $h=1 / 2$, since $C_{\delta_{1}}=1$. The latter value agrees precisely with the ground state energy of the corresponding affine representation when $k=1$ as given by (4.13).

We want to go a little further and recover states in the $R=1$ model whose weight is one above the ground states. Let us pick, for example, a multiplet that transforms on the representation $\Lambda=3 \delta_{1}$. In the large radius limit, this representation arises for the first time among the states of weight $h=3$. In fact, in eq. (4.1) terms containing $y_{1}^{3}$ are multiplied by $q^{3}$ or higher powers of $q$. Since $C_{3 \delta_{1}}=3$, the proposal (4.9) tells us that the weight of this multiplet gets deformed to $h=3-\frac{3}{2}=\frac{3}{2}$. Hence, it should appear among the first descendants of the sector over the fundamental representation. Indeed, the irreducible representation with highest weight $3 \delta_{1}$ is contained in the tensor product of the fundamental representation with the adjoint representation. Thus, $Z_{B ; S}^{\mathrm{FF}}$ contains this representation with $h=\frac{3}{2}$ exactly as predicted by eq. (4.9).

\section{Conclusions, open questions and outlook}

This work contains two central results. To begin with, we have been able to compute the exact boundary spectrum of a volume filling brane on the 3-dimensional supersphere $S^{3 \mid 2}$ for all values of the curvature radius $R$. With a little bit of extra work it should be possible to extend our formulas to higher dimensional superspheres and also to other spectra, including the spectrum of the bulk fields (see comments below). The second result concerns the duality between the supersphere PCM and the osp $(2 S+2 \mid 2 S)$ GN model. More specifically, we were able identify the spectrum at the special point $R=1$ with that of a free field theory, namely of the model (1.5) with a particular choice of boundary conditions. This is consistent with a recent conjecture in [24] and it provides extremely strong additional support for the duality.

The supersphere $S^{3 \mid 2}$ and its higher dimensional generalizations have been advocated in the past [20, 21] as good toy models for the world-sheet description of string theory on $A d S_{5} \times S^{5}$. Obviously, the defining equations for both $A d S_{5}$ and $S^{5}$ are very similar to our basic constraint (1.1). What is more important, however, is that the world-sheet models for $A d S_{5} \times S^{5}=[\mathrm{PSU}(2,2 \mid 4) / \mathrm{SO}(1,4) \times \mathrm{SO}(5)]_{0}$ and the supersphere theory give rise to continuous families of $2 \mathrm{D}$ conformal field theories with many common features. In both cases, the non-abelian global symmetries remain unbroken. On the other hand, they are 
not enhanced into affine symmetries, at least not for generic points in the moduli space. Consequently, it seems reasonable to speculate briefly about possible lessons the supersphere models might teach us for the world-sheet descriptions of string theory in $A d S_{5} \times S^{5} 9$

To begin with, it is certainly possible to determine the exact spectrum of the free sigma model on the supercoset $\mathrm{PSU}(2,2 \mid 4) / \mathrm{SO}(1,4) \times \mathrm{SO}(5)$ at $R=\infty$, much as this was done here for the supersphere. The deformation of the spectrum away from $R=\infty$ cannot be as simple as in the supersphere case. In fact, we know for sure that there are some operators whose anomalous dimensions do not possess a quasi-abelian dependence of the radius $R$ (or the 't Hooft coupling). It might be interesting, however, to study whether there is some subset of operators whose dimensions are given by eq. (1.8). Since we have nothing to say about this right now, let us just imagine that in some way we were able to deform the entire spectrum. Then we could start to look for special values of the radius $R$ at which the spectrum contains half-integer or integer values only. We know for sure that such a point exists, namely the radius $R_{0}$ for with the string model becomes dual to the free $N=4$ supersymmetric Yang-Mills theory. One might hope that such a point is described by a free world-sheet theory, just as it is the case for the superspheres. In this sense, the dual of the free Yang-Mills theory would be the analogue of the free GN model. If one found such strong-weak coupling duality within the world-sheet description of strings in $A d S$, it would reduce the AdS/CFT correspondence to a remaining weak-weak coupling duality. Worldsheet descriptions of weakly coupled gauge theory have appeared in the literature, see e.g. 37, 38. or the recent work [39] for two developments that seem relevant for what we have just outlined.

Finding an explicit action for such a free world-sheet model and its deformation might have two interesting applications. To begin with, it could provide a better starting point for the quantization of the string theory on $A d S_{5} \times S^{5}$. In fact, let us point out that our $\operatorname{OSP}(2 S+2 \mid 2 S)$-GN model is much simpler than the original supersphere PCM: While the perturbative expansion of the latter contains terms of any order in the basic fields, the former has no terms beyond fourth order. Furthermore, the perturbative expansion for the conjectured weakly coupled dual of the strongly coupled $A d S_{5} \times S^{5}$ sigma model could be compared order by order to the perturbative expansion in the gauge theory, see again [39. One might even hope to prove the AdS/CFT duality using such an intermediate world-sheet model. Of course all this remains mere speculation for now. In particular, it is clear that our analysis of supersphere models exploited compactness of the target's bosonic base. More work is necessary to include non-compact targets such as $A d S_{5} \times S^{5}$ or $A d S_{4} \times \mathbb{C P}^{3}$.

After all these comments on possible implications for the AdS/CFT correspondence, we would like to close with a few remarks on the bulk spectrum of the supersphere models. The analysis of boundary deformations in 22 puts much stress on the fact that computations where only possible for very particular boundary spectra. In fact, open strings had to be localized at one point in a background in order to avoid running into mixing problems. For the

\footnotetext{
${ }^{9}$ Similar remarks apply obviously to $A d S_{4} \times \mathbb{C P}^{3}$.
} 
superspheres, similar issues do not arise. While 22] focused on a bulk deformation preserving global left and right transformations simultaneously, the current-current perturbation (1.6) considered here is of a very different type. Since the deforming operator does not involve any tachyonic vertex operators, there is no mixing problem, neither for boundary theories, nor even for the bulk. On the other hand, the perturbation breaks the global bulk symmetry down to a single diagonal action of the symmetry algebra. Therefore, it should be possible to deform bulk spectra, but it might be more difficult to identify the relevant osp $(2 S+2 \mid 2 S)$ action as we deform from $R=1$ to $R=\infty$. We will return to these issues in a future publication.

Acknowledgments: We would like to thank Thomas Creutzig, Guiliano Niccoli, Peter Rønne, Jörg Teschner, Alexei Tsvelik and in particular Constantin Candu and Hubert Saleur for numerous stimulating discussion and many useful comments. T.Q. and V.S. are also grateful for the kind hospitality at the Isaac Newton Institute and the inspiring atmosphere during its Workshop "Strong Fields, Integrability and Strings". The research of T.Q. is funded by a Marie Curie Intra-European Fellowship, contract number MEIF-CT-2007-041765. We furthermore acknowledge partial support from the EU Research Training Network Superstring theory, MRTN-CT-2004-512194 and from ForcesUniverse, MRTN-CT-2004-005104. 


\section{A Some aspects of the representation theory of $\operatorname{OSP}(4 \mid 2)$}

Our first appendix contains a number of basic notations and results concerning the Lie superalgebra osp $(4 \mid 2)$. These are used frequently in the main text. The complex superalgebra $g:=\operatorname{osp}(4 \mid 2)$ may be realized as the set of matrices

$$
\operatorname{osp}(4 \mid 2)=\left\{\left(\begin{array}{cc}
A & B \\
J_{2} B^{t} & D
\end{array}\right): A^{t}=-A \text { and } D^{t} J_{2}=-J_{2} D\right\}
$$

with $J_{2}=\left(\begin{array}{cc}0 & -1 \\ 1 & 0\end{array}\right)$ and the standard definition of graded commutators. We have the usual separation of the superalgebra into a bosonic $g_{\overline{0}}=\operatorname{sp}(2) \oplus \operatorname{so}(4) \cong \operatorname{sl}(2) \oplus \operatorname{sl}(2) \oplus \operatorname{sl}(2)$ and a fermionic $g_{\overline{1}}$ subspace. In addition, the superalgebra has a $\mathbb{Z}$-grading that is compatible with its $\mathbb{Z}_{2}$ structure, i.e. $g=g_{-2} \oplus g_{-1} \oplus g_{0} \oplus g_{1} \oplus g_{2}$, where the relation $\left[g_{i}, g_{j}\right]=g_{i+j}$ holds, with $g_{0} \cong \mathrm{so}(4) \oplus \operatorname{gl}(1), g_{\overline{0}}=g_{-2} \oplus g_{0} \oplus g_{2}$ and $g_{\overline{1}}=g_{-1} \oplus g_{1}$.

An integral dominant highest weight $\Lambda=\left(j_{1}, j_{2}, j_{3}\right)$ of $g_{\overline{0}}$ is also one for the full superalgebra $g$ if it obeys the consistency conditions:

$$
j_{1}=0 \Rightarrow j_{2}=j_{3}=0 \quad, \quad j_{1}=\frac{1}{2} \Rightarrow j_{2}=j_{3}
$$

where the first spin is related to the symplectic subalgebra and the two others to the orthogonal one. The finite dimensional irreducible representations $[\Lambda]$ of $g$ are constructed as follows. Taking an irreducible highest weight representation $(\Lambda)$ of $g_{0} \cong \operatorname{so}(4) \oplus \operatorname{gl}(1)$ with highest weight $\Lambda=\left(j_{1}, j_{2}, j_{3}\right)$ associated to the highest weight vector $v_{\Lambda}$, we set

$$
M_{\Lambda}=U(g)\left(E_{1}^{-}\right)^{2 j_{1}+1} v_{\Lambda}, \quad K_{\Lambda}=\left(\operatorname{Ind}_{p}^{g}(\Lambda)\right) / M_{\Lambda}
$$

where $U(g)$ is the universal enveloping algebra of $g, E_{1}^{-}$is the lowering operator of the symplectic subalgebra and $p=g_{0} \oplus g_{1} \oplus g_{2}$. In the above equation, we have considered the $g_{0}$-module $(\Lambda)$ as a $p$-module by letting $g_{i}, i=1,2$ act trivially on it. The finite dimensional representation $K_{\Lambda}$ is called the Kac module of $\Lambda$ and is generically irreducible. The set of Kac modules is divided into typical and atypical ones. If the Kac module $K_{\Lambda}$ is typical, then it is guaranteed to be irreducible. In this case we define the simple module $[\Lambda]$ to be $K_{\Lambda}$. If, however, one or more of the following atypicality conditions

$$
\begin{aligned}
& 2 j_{1}=-j_{2}-j_{3}, \\
& 2 j_{1}=j_{2}+j_{3}+2, \\
& 2 j_{1}= \pm\left(j_{2}-j_{3}\right)+1
\end{aligned}
$$

hold, then $K_{\Lambda}$ is atypical and will generically contain a maximal invariant subspace $I_{\Lambda}$ without being fully reducible, i.e. it will contain indecomposable constituents. In those cases, we set $[\Lambda]=K_{\Lambda} / I_{\Lambda}$. It can occur however that $I_{\Lambda}=0$ even though $K_{\Lambda}$ is atypical.

The eigenvalue of the quadratic Casimir in the simple module $[\Lambda]$ is given by the formula

$$
C_{2}(\Lambda)=-4 j_{1}\left(j_{1}-1\right)+2 j_{2}\left(j_{2}+1\right)+2 j_{3}\left(j_{3}+1\right) .
$$


In particular, $C_{2}(\Lambda)$ is always a square, i.e. $C_{2}(\Lambda)=k^{2}, k \in \mathbb{N}$, on atypical representations $[\Lambda]$. The atypical weights $\Lambda=\left(j_{1}, j_{2}, j_{3}\right)$ can be divided into blocks $\Gamma_{k}$, such that weights in $\Gamma_{k}$ possess the same eigenvalue $C_{2}(\Lambda)=k^{2}$ of the quadratic Casimir element. The corresponding atypical labels can be listed explicitly [40],

$$
\begin{aligned}
& \Gamma_{0}=\left\{\lambda_{0,0}=(0,0,0), \lambda_{0, l}=\frac{1}{2}(l+1, l-1, l-1), l \geq 1\right\} \\
& \Gamma_{k}=\left\{\lambda_{k, l}, l \in \mathbb{Z}\right\}
\end{aligned}
$$

where

$$
\lambda_{k, l}=\left\{\begin{array}{lr}
\frac{1}{2}(-l+2,-l-k,-l+k) & \text { if } l \leq-k \\
\frac{1}{2}(-l+1, l+k-1,-l+k-1) & \text { if }-k+1 \leq l \leq 0 \\
\frac{1}{2}(l+1, l+k-1,-l+k-1) & \text { if } 0 \leq l \leq k-1 \\
\frac{1}{2}(l+2, l+k, l-k) & \text { if } k \leq l
\end{array} .\right.
$$

One sees easily, that the weights $\lambda_{k,-l}$ for $k \geq 1$ may be obtained from $\lambda_{k, l}$ by simply exchanging the second and the third Dynkin label. Furthermore, it is possible to distinguish the weights $\lambda_{k, l}$ according to the atypicality condition (A.2) they obey. The only weight to fulfill the first condition is $\lambda_{0,0}$. The weights belonging to the second condition are $\lambda_{0, l}$ for $l \geq 1$ and $\lambda_{k, \pm l}$ for $l \geq k$. Finally, those the satisfy the last atypicality relation are the $\lambda_{k, \pm l}$ for $l<k$.

The only atypical Kac modules $K\left(\lambda_{k, l}\right)$ which are irreducible correspond to the weights $\lambda_{k, 0}$ for $k \geq 0$ and to $\lambda_{0,1}$. The indecomposable structure of the remaining ones can be deciphered from the following diagram,

$$
\begin{aligned}
K_{\lambda_{0,2}}: & {\left[\lambda_{0,2}\right] \longrightarrow\left[\lambda_{0,0}\right] \oplus\left[\lambda_{0,1}\right] } \\
K_{\lambda_{0, l}}: & {\left[\lambda_{0, l}\right] \longrightarrow\left[\lambda_{0, l-1}\right] \text { for } l \geq 3 } \\
K_{\lambda_{k, l}}: & {\left[\lambda_{k, l}\right] \longrightarrow\left[\lambda_{k, l-1}\right] \text { for } l \geq 1 } \\
K_{\lambda_{k, l}}: & {\left[\lambda_{k, l}\right] \longrightarrow\left[\lambda_{k, l+1}\right] \text { for } l \leq-1 . }
\end{aligned}
$$

The dimension of the typical Kac modules is

$$
\operatorname{dim}\left[K_{\left(j_{1}, j_{2}, j_{3}\right)}\right]=16\left(2 j_{1}-1\right)\left(2 j_{2}+1\right)\left(2 j_{3}+1\right)
$$

whereas the dimension of the atypical ones may be inferred from their structure, together with the following formulas for the dimension of the irreducible representations,

$$
\begin{aligned}
\operatorname{dim}\left[\lambda_{0,0}\right] & =1, \quad \operatorname{dim}\left[\lambda_{0,1}\right]=17, \quad \operatorname{dim}\left[\lambda_{k, 0}\right]=4 k^{2}+2 \\
\operatorname{dim}\left[\lambda_{0, l}\right] & =(2 l+1)\left[(2 l+1)^{2}-3\right] \text { for } l \geq 2 \\
\operatorname{dim}\left[\lambda_{k, l}\right] & =(2 l+1)\left[4\left(k^{2}-1\right)-(2 l+1)^{2}+7\right] \text { for } l \leq k-1 \\
\operatorname{dim}\left[\lambda_{k, l}\right] & =(2 l+3)\left[(2 l+3)^{2}-4\left(k^{2}-1\right)-7\right] \text { for } l \geq k
\end{aligned}
$$

where, of course, $\operatorname{dim}\left[\lambda_{k,-l}\right]=\operatorname{dim}\left[\lambda_{k, l}\right]$. The decomposition of $K_{\Lambda}$ for $j_{1} \geq 1$, whether typical or not, into irreducible modules of the bosonic subalgebra has been computed in 41 . 
It takes the form

$$
\begin{aligned}
{\left[K_{\Lambda}\right]_{g_{\overline{0}}} \cong } & \left(j_{1}, j_{2}, j_{3}\right) \bigoplus_{\alpha, \beta= \pm \frac{1}{2}}\left(j_{1}-\frac{1}{2}, j_{2}+\alpha, j_{3}+\beta\right) \\
& \bigoplus_{\alpha= \pm 1}\left[\left(j_{1}-1, j_{2}+\alpha, j_{3}\right) \oplus\left(j_{1}-1, j_{2}, j_{3}+\alpha\right)\right] \oplus 2\left(j_{1}-1, j_{2}, j_{3}\right) \\
& \oplus \bigoplus_{\alpha, \beta= \pm \frac{1}{2}}\left(j_{1}-\frac{3}{2}, j_{2}+\alpha, j_{3}+\beta\right) \oplus\left(j_{1}-2, j_{2}, j_{3}\right) .
\end{aligned}
$$

There are a few special cases for which the decomposition is not generic. If $j_{1} \leq 2, j_{2} \leq 1$ or $j_{3} \leq 1$ then the above decomposition formula must be truncated at the point where one ore more of the labels become negative. Moreover, there are two cases for which the multiplicity of the $\left(j_{1}-1, j_{2}, j_{3}\right)$ submodule has to be changed. If $j_{1}=1, j_{2}>0, j_{3}>0$ or $j_{1}>1, j_{2}=0, j_{3}>0$ or $j_{1}>1, j_{2}>0, j_{3}=0$, then this block will appear only once and if both $j_{2}$ and $j_{3}$ are null, then it will not be present at all.

When $j_{1}=\frac{1}{2}$, the Kac modules $K_{\Lambda}$ with weight $\Lambda$ obeying the consistency conditions (A.1) are equal to the irreducible modules $\left[\frac{1}{2}, \frac{k}{2}, \frac{k}{2}\right]$ and they possess the following structure

$$
\left[\frac{1}{2}, \frac{k}{2}, \frac{k}{2}\right]_{\mid g_{\overline{0}}} \cong\left(\frac{1}{2}, \frac{k}{2}, \frac{k}{2}\right) \oplus\left(0, \frac{k+1}{2}, \frac{k+1}{2}\right) \oplus\left(0, \frac{k-1}{2}, \frac{k-1}{2}\right) .
$$

Finally, the Kac module $K_{[0,0,0]}$ is trivial.

\section{B Some useful identities}

In this appendix we collect a few definitions and identities that we have employed to obtain the Casimir decompositions in sections 2.3 and 3.3. We also provide the first few terms in the Casimir decomposition of the partition function $Z_{B}^{\mathrm{FF}}$ for $S=1$.

\section{B.1 Identities used in the Casimir decomposition}

To begin with, let us briefly recall the definition of Jacobi's $\theta$ functions. In our conventions they are given by

$$
\begin{aligned}
& \theta_{1}(q \mid z)=-i \sum_{r \in \mathbb{Z}+\frac{1}{2}}(-1)^{r-\frac{1}{2}} z^{r} q^{\frac{r^{2}}{2}}=-i z^{\frac{1}{2}} q^{\frac{1}{8}} \prod_{n=1}^{\infty}\left(1-q^{n}\right)\left(1-z q^{n}\right)\left(1-z^{-1} q^{n-1}\right) \\
& \theta_{2}(q \mid z)=\sum_{r \in \mathbb{Z}+\frac{1}{2}} z^{r} q^{\frac{r^{2}}{2}}=z^{\frac{1}{2}} q^{\frac{1}{8}} \prod_{n=1}^{\infty}\left(1-q^{n}\right)\left(1+z q^{n}\right)\left(1+z^{-1} q^{n-1}\right) \\
& \theta_{3}(q \mid z)=\sum_{r \in \mathbb{Z}} z^{r} q^{\frac{r^{2}}{2}}=\prod_{n=1}^{\infty}\left(1-q^{n}\right) \prod_{r \in \mathbb{N}+\frac{1}{2}}\left(1+z q^{r}\right)\left(1+z^{-1} q^{r}\right) \\
& \theta_{4}(q \mid z)=\sum_{r \in \mathbb{Z}}(-1)^{r} z^{r} q^{\frac{r^{2}}{2}}=\prod_{n=1}^{\infty}\left(1-q^{n}\right) \prod_{r \in \mathbb{N}+\frac{1}{2}}\left(1-z q^{r}\right)\left(1-z^{-1} q^{r}\right)
\end{aligned}
$$


The following two lemmata contain auxiliary formulas that are needed to rewrite the partition function (2.16) in terms of characters of osp(4|2).

Lemma B.1.

$$
\prod_{n=1}^{\infty} \frac{1}{\left(1-z q^{n}\right)\left(1-z^{-1} q^{n}\right)}=\sum_{n \in \mathbb{Z}} z^{n} \sum_{m=0}^{\infty}(-1)^{m} \frac{q^{\frac{m}{2}(m+2 n+1)}-q^{\frac{m}{2}(m+2 n-1)}}{\phi(q)^{2}} .
$$

Proof. We assume that $|q|<|z|<1$, which is the relevant condition for the above expansion to make sense. We want to find the coefficients $f_{l}^{N}(q)$ in the relation

$$
\sum_{l \in \mathbb{Z}} f_{l}^{N}(q) z^{l}=\frac{1}{(1-z) \prod_{n=1}^{N}\left(1-z q^{n}\right)\left(1-z^{-1} q^{n}\right)} .
$$

To do this, we multiply both sides by $z^{-k-1}$ and integrate them over $z$ along a contour that surrounds zero in a counterclockwise direction. In order to stay within the region $|z|<1$ it must cling to the unit circle on the inside. The left hand side of the previous equation gives us the coefficient $f_{k}^{N}(q)$. The right hand side is zero for $z=0$ and the first order poles that are encircled by the contour are at $z=q^{n}$ for $n=1, \ldots, N$. Their residues are given by

$$
\lim _{z \rightarrow q^{n}} \frac{z^{-k-1}\left(z-q^{n}\right)}{(1-z) \prod_{l=1}^{N}\left(1-z q^{l}\right)\left(1-z^{-1} q^{l}\right)}=\frac{(-1)^{n-1} q^{\frac{n}{2}(n-2 k-1)}}{\prod_{l=1}^{N+n}\left(1-q^{l}\right) \prod_{l=1}^{N-n}\left(1-q^{l}\right)} .
$$

If we finally remove our cutoff $N$ by sending $N \rightarrow \infty$ we arrive at

$$
\frac{1}{(1-z) \prod_{n=1}^{\infty}\left(1-z q^{n}\right)\left(1-z^{-1} q^{n}\right)}=\sum_{k \in \mathbb{Z}} z^{k} \sum_{n=0}^{\infty} \frac{(-1)^{n-1} q^{\frac{n}{2}(n-1-2 k)}}{\phi(q)^{2}} .
$$

Multiplying both sides by $1-z$ and using the lemma B.2 below to shuffle some minus signs around completes the proof.

\section{Lemma B.2.}

$$
\begin{aligned}
& \sum_{m=1}^{2 n}(-1)^{m} q^{\frac{m(m-1)}{2}-m n}=0 \quad \text { for } \quad n \geq 1 \\
& \sum_{m=1}^{\infty} \sum_{s=-r}^{r}(-1)^{m} q^{\frac{m(m-1)}{2}-m(n+s)}\left(1-q^{m}\right)=\sum_{m=1}^{\infty} \sum_{s=-r}^{r}(-1)^{m} q^{\frac{m(m-1)}{2}-m(-n+s)}\left(1-q^{m}\right) .
\end{aligned}
$$

Proof. The first equation is shown to be true by splitting the sum in $\sum_{m=1}^{n}$ and $\sum_{m=n+1}^{2 n}$ and showing that they are equal up to a sign. The second equation then follows easily from the first.

There are a number of very simple auxiliary formulas that are needed for the Casimir decomposition in section 2.3. Let us only list two of them here

$$
\sum_{r=0}^{\infty}(-1)^{r} q^{\frac{r(r+2)}{4}}\left(1-q^{r+2}\right) a_{r}=\sum_{r=0}^{\infty}(-1)^{r} q^{\frac{r(r+2)}{4}}\left(a_{r}-a_{r-2}\right)
$$




$$
\begin{aligned}
& \left(q^{\left(j_{2}-\frac{r}{2}\right)^{2}}-q^{\left(j_{2}+\frac{r}{2}+1\right)^{2}}\right)\left(q^{\left(j_{3}-\frac{r}{2}\right)^{2}}-q^{\left(j_{3}+\frac{r}{2}+1\right)^{2}}\right)=q^{j_{2}\left(j_{2}+1\right)+j_{3}\left(j_{3}+1\right)} q^{\frac{r^{2}}{2}+r+1} \\
& \quad \times\left(q^{-(r+1)\left(j_{2}+j_{3}+1\right)}+q^{(r+1)\left(j_{2}+j_{3}+1\right)}-q^{(r+1)\left(j_{2}-j_{3}\right)}-q^{-(r+1)\left(j_{2}-j_{3}\right)}\right)
\end{aligned}
$$

\section{B.2 Casimir decomposition of $Z_{B}^{\mathrm{FF}}$}

In section 3.3 we obtained closed formulas (3.24) and (3.26) for the Casimir decomposition of the partition function $Z_{B}^{\mathrm{FF}}$. Since our expression for the branching functions is a bit complicated, let us reproduce the first few terms of the partition function explicitly,

$$
\begin{aligned}
& Z_{B ; S=1}^{\mathrm{FF}}(q)=q^{0} \chi_{[0,0,0]}+q^{\frac{1}{2}} \chi_{\left[\frac{1}{2}, 0,0\right]}+q^{1} \chi_{[1,0,0]}+q^{\frac{3}{2}}\left(\chi_{\left[\frac{3}{2}, 0,0\right]}+\chi_{\left[\frac{1}{2}, 0,0\right]}\right) \\
& +q^{2}\left(\chi_{[2,0,0]}+\chi_{[1,0,0]}+\chi_{\left[\frac{1}{2}, \frac{1}{2}, \frac{1}{2}\right]}+\chi_{[0,0,0]}\right) \\
& +q^{\frac{5}{2}}\left(\chi_{\left[\frac{5}{2}, 0,0\right]}+\chi_{\left[\frac{3}{2}, 0,0\right]}+\chi_{\left[1, \frac{1}{2}, \frac{1}{2}\right]}+2 \chi_{\left[\frac{1}{2}, 0,0\right]}\right) \\
& +q^{3}\left(\chi_{[3,0,0]}+\chi_{[2,0,0]}+\chi_{\left[\frac{3}{2}, \frac{1}{2}, \frac{1}{2}\right]}+4 \chi_{[1,0,0]}+\chi_{\left[\frac{1}{2}, \frac{1}{2}, \frac{1}{2}\right]}+\chi_{[0,0,0]}\right) \\
& +q^{\frac{7}{2}}\left(\chi_{\left[\frac{7}{2}, 0,0\right]}+\chi_{\left[\frac{5}{2}, 0,0\right]}+\chi_{\left[2, \frac{1}{2}, \frac{1}{2}\right]}+3 \chi_{\left[\frac{3}{2}, 0,0\right]}+2 \chi_{\left[1, \frac{1}{2}, \frac{1}{2}\right]}+3 \chi_{\left[\frac{1}{2}, 0,0\right]}\right) \\
& +q^{4}\left(\chi_{[4,0,0]}+\chi_{[3,0,0]}+\chi_{\left[\frac{5}{2}, \frac{1}{2}, \frac{1}{2}\right]}+3 \chi_{[2,0,0]}+2 \chi_{\left[\frac{3}{2}, \frac{1}{2}, \frac{1}{2}\right]}+\chi_{[1,1,0]}+\chi_{[1,0,1]}\right. \\
& \left.+6 \chi_{[1,0,0]}+4 \chi_{\left[\frac{1}{2}, \frac{1}{2}, \frac{1}{2}\right]}+3 \chi_{[0,0,0]}\right) \\
& +q^{\frac{9}{2}}\left(\chi_{\left[\frac{9}{2}, 0,0\right]}+\chi_{\left[\frac{7}{2}, 0,0\right]}+\chi_{\left[3, \frac{1}{2}, \frac{1}{2}\right]}+3 \chi_{\left[\frac{5}{2}, 0,0\right]}+2 \chi_{\left[2, \frac{1}{2}, \frac{1}{2}\right]}+\chi_{\left[\frac{3}{2}, 1,0\right]}\right. \\
& \left.+\chi_{\left[\frac{3}{2}, 0,1\right]}+5 \chi_{\left[\frac{3}{2}, 0,0\right]}+4 \chi_{\left[1, \frac{1}{2}, \frac{1}{2}\right]}+\chi_{\left[\frac{1}{2}, 1,1\right]}+7 \chi_{\left[\frac{1}{2}, 0,0\right]}\right) \\
& +q^{5}\left(\chi_{[5,0,0]}+\chi_{[4,0,0]}+\chi_{\left[\frac{7}{2}, \frac{1}{2}, \frac{1}{2}\right]}+3 \chi_{[3,0,0]}+2 \chi_{\left[\frac{5}{2}, \frac{1}{2}, \frac{1}{2}\right]}+\chi_{[2,1,0]}+\chi_{[2,0,1]}\right. \\
& \left.+5 \chi_{[2,0,0]}+5 \chi_{\left[\frac{3}{2}, \frac{1}{2}, \frac{1}{2}\right]}+\chi_{[1,1,1]}+\chi_{[1,1,0]}+\chi_{[1,0,1]}+14 \chi_{[1,0,0]}+5 \chi_{\left[\frac{1}{2}, \frac{1}{2}, \frac{1}{2}\right]}+3 \chi_{[0,0,0]}\right) \\
& +q^{\frac{11}{2}}\left(\chi_{\left[\frac{11}{2}, 0,0\right]}+\chi_{\left[\frac{9}{2}, 0,0\right]}+\chi_{\left[4, \frac{1}{2}, \frac{1}{2}\right]}+3 \chi_{\left[\frac{7}{2}, 0,0\right]}+2 \chi_{\left[3, \frac{1}{2}, \frac{1}{2}\right]}+\chi_{\left[\frac{5}{2}, 1,0\right]}\right. \\
& +\chi_{\left[\frac{5}{2}, 0,1\right]}+5 \chi_{\left[\frac{5}{2}, 0,0\right]}+5 \chi_{\left[2, \frac{1}{2}, \frac{1}{2}\right]}+10 \chi_{\left[\frac{3}{2}, 0,0\right]}+2 \chi_{\left[\frac{3}{2}, 1,0\right]}+2 \chi_{\left[\frac{3}{2}, 0,1\right]}+\chi_{\left[\frac{3}{2}, 1,1\right]} \\
& \left.+8 \chi_{\left[1, \frac{1}{2}, \frac{1}{2}\right]}+\chi_{\left[\frac{1}{2}, 1,1\right]}+11 \chi_{\left[\frac{1}{2}, 0,0\right]}\right) \\
& +q^{6}\left(\chi_{[6,0,0]}+\chi_{[5,0,0]}+\chi_{\left[\frac{9}{2}, \frac{1}{2}, \frac{1}{2}\right]}+3 \chi_{[4,0,0]}+2 \chi_{\left[\frac{7}{2}, \frac{1}{2}, \frac{1}{2}\right]}+\chi_{[3,1,0]}\right. \\
& +\chi_{[3,0,1]}+5 \chi_{[3,0,0]}+5 \chi_{\left[\frac{5}{2}, \frac{1}{2}, \frac{1}{2}\right]}+11 \chi_{[2,0,0]}+2 \chi_{[2,1,0]}+2 \chi_{[2,0,1]}+\chi_{[2,1,1]} \\
& \left.+11 \chi_{\left[\frac{3}{2}, \frac{1}{2}, \frac{1}{2}\right]}+2 \chi_{[1,1,1]}+4 \chi_{[1,1,0]}+4 \chi_{[1,0,1]}+22 \chi_{[1,0,0]}+13 \chi_{\left[\frac{1}{2}, \frac{1}{2}, \frac{1}{2}\right]}+9 \chi_{[0,0,0]}\right) \\
& +q^{\frac{13}{2}}\left(\chi_{\left[\frac{13}{2}, 0,0\right]}+\chi_{\left[\frac{11}{2}, 0,0\right]}+\chi_{\left[5, \frac{1}{2}, \frac{1}{2}\right]}+3 \chi_{\left[\frac{9}{2}, 0,0\right]}+2 \chi_{\left[4, \frac{1}{2}, \frac{1}{2}\right]}+\chi_{\left[\frac{7}{2}, 1,0\right]}\right. \\
& +\chi_{\left[\frac{7}{2}, 0,1\right]}+5 \chi_{\left[\frac{7}{2}, 0,0\right]}+5 \chi_{\left[3, \frac{1}{2}, \frac{1}{2}\right]}+11 \chi_{\left[\frac{5}{2}, 0,0\right]}+2 \chi_{\left[\frac{5}{2}, 1,0\right]}+2 \chi_{\left[\frac{5}{2}, 0,1\right]}+\chi_{\left[\frac{5}{2}, 1,1\right]} \\
& +11 \chi_{\left[2, \frac{1}{2}, \frac{1}{2}\right]}+2 \chi_{\left[\frac{3}{2}, 1,1\right]}+5 \chi_{\left[\frac{3}{2}, 1,0\right]}+5 \chi_{\left[\frac{3}{2}, 0,1\right]}+16 \chi_{\left[\frac{3}{2}, 0,0\right]}+15 \chi_{\left[1, \frac{1}{2}, \frac{1}{2}\right]}+\chi_{\left[1, \frac{3}{2}, \frac{1}{2}\right]} \\
& \left.+\chi_{\left[1, \frac{1}{2}, \frac{3}{2}\right]}+4 \chi_{\left[\frac{1}{2}, 1,1\right]}+21 \chi_{\left[\frac{1}{2}, 0,0\right]}\right)+\ldots .
\end{aligned}
$$

One may deform this expression to values $R \neq 1$ by means of the formula (3.35) at the end of section 3.4. 


\section{Recombination of the bosonic characters}

Let $Z$ be a partition function with $\operatorname{osp}(4 \mid 2)$ symmetry. If we denote the characters of the bosonic subalgebra by $\chi_{\left(j_{1}, j_{2}, j_{3}\right)}^{B}\left(z_{i}\right)=\chi_{j_{1}}\left(z_{1}\right) \chi_{j_{2}}\left(z_{2}\right) \chi_{j_{3}}\left(z_{3}\right)$, we can write the partition function as

$$
Z=\sum_{\lambda \in \mathcal{J}} \chi_{\lambda}^{B}\left(z_{1}, z_{2}, z_{3}\right) \psi_{\lambda}^{B}(q)=\sum_{\lambda \in \mathcal{J}^{\prime}} \chi_{\lambda}^{K}\left(z_{1}, z_{2}, z_{3}\right) \psi_{\lambda}^{K}(q)
$$

where $\mathcal{J}^{\prime} \subset \mathcal{J}$ is the set of labels in $\mathcal{J}=\left\{\left(j_{1}, j_{2}, j_{3}\right) ; j_{i}=0,1 / 2,1,3 / 2, \ldots\right\}$ that are compatible with the consistency conditions (A.1). Here, the first decomposition is in terms of bosonic characters while the second one is based on the characters of Kac modules. In order to find the relations between these two decompositions, we recall that the roots of the four fermionic lowering operators in $g_{-1}:=\operatorname{osp}(4 \mid 2)_{-1}$ are

$$
\alpha_{1}=\left(-\frac{1}{2}, \frac{1}{2}, \frac{1}{2}\right) \alpha_{2}=\left(-\frac{1}{2}, \frac{1}{2},-\frac{1}{2}\right) \alpha_{3}=\left(-\frac{1}{2},-\frac{1}{2}, \frac{1}{2}\right) \alpha_{4}=\left(-\frac{1}{2},-\frac{1}{2},-\frac{1}{2}\right) .
$$

Let us first discuss the generic label $\lambda=\left(j_{1}, j_{2}, j_{3}\right)$ where either $j_{1} \geq \frac{3}{2}$, or $j_{1}=1$ and $\left(j_{2}, j_{3}\right) \neq(0,0)$. In such cases we can write the decomposition of the Kac module character $\chi_{\lambda}^{K}$ as

$$
\chi_{\lambda}^{K}=\sum_{i=0}^{4} \sum_{\beta \in \Lambda^{i}\left(g_{-1}\right)} \chi_{\lambda+\beta}^{B}
$$

where $\beta$ is any of the weights that appear in the $i^{\text {th }}$ exterior product $\Lambda^{i}\left(g_{-1}\right)$ of $g_{-1}$. We also allow for negative spins using the formal prescription $\chi_{j}=-\chi_{-j-1}$. To treat the remaining cases with $j_{1} \leq \frac{1}{2}$ we employ the formulas developed in appendix A. Inserting the decomposition of Kac modules into the partition function $Z$ leads to a formula that expresses the bosonic branching functions $\psi_{\lambda}^{B}$ as sums of the branching functions $\psi_{\mu}^{K}$. Our main aim is to invert this relation, i.e. to determine the branching functions $\psi^{K}$ in terms of $\psi^{B}$. To this end let us state a few basic properties of $\psi^{K}$ that will be checked afterwards, once we have an explicit formula,

$$
\psi_{\left[j_{1}, j_{2}, j_{3}\right]}^{K}=-\psi_{\left[j_{1},-j_{2}-1, j_{3}\right]}^{K}=-\psi_{\left[j_{1}, j_{2},-j_{3}-1\right]}^{K} .
$$

If we take this behavior of $\psi^{K}$ for granted the decomposition formulas for the partition function $Z$ and of $\chi^{K}$ in terms of bosonic characters imply,

$$
\psi_{\lambda}^{B}=\sum_{i=0}^{4} \sum_{\beta \in \Lambda^{i}\left(g_{-1}\right)} \psi_{\lambda-\beta}^{K}
$$

for all $\lambda \in \mathcal{J}^{\prime}$. Inverting this expression leads to the following result

$$
\psi_{\Lambda}^{K}=\sum_{n=0}^{\infty}(-1)^{n} \sum_{\beta \in \operatorname{Sym}^{n}\left(g_{-1}\right)} \psi_{\Lambda-\beta}^{B} .
$$


To establish formula (C.6) we plug (C.5) into (C.6). Thereby we obtain

$$
\psi_{\Lambda}^{K}=\sum_{i=0}^{\infty}(-1)^{i} \underbrace{\sum_{j=0}^{4}(-1)^{j} \sum_{\beta \in \operatorname{Sym}^{i-j}\left(g_{-1}\right)} \sum_{\gamma \in \Lambda^{j}\left(g_{-1}\right)} \psi_{\Lambda-\beta-\gamma}^{K}}_{=0 \text { if } i \neq 0}=\psi_{\Lambda}^{K},
$$

thus showing that (C.6) inverts (C.5). In (C.7) we have set $\operatorname{Sym}^{n}(V)=\emptyset$ if $n<0$ and used the identity:

$$
\sum_{j=0}^{4}(-1)^{j} \sum_{\beta \in \operatorname{Sym}^{i-j}(V)} \sum_{\gamma \in \Lambda^{j}(V)} c(\beta+\gamma)=0,
$$

which is true for every four dimensional vector space $V$ and every function $c$ as long as $i \geq 1$. To show (C.8), we introduce the symbol $\ominus$ which is to be understood as a sort of a negative of a direct sum as for example in $A \oplus B \ominus B=A$. Then (C.8) is equivalent to $\bigoplus_{j=0}^{4} \ominus^{j} \operatorname{Sym}^{i-j}(V) \otimes \Lambda^{j}(V)=0$ if $i \geq 1$, which can be shown using standard Young tableaux techniques. Denote a tableau consisting of one single row with $m$ boxes by $1^{m}$ and a tableau with one single column of $n$ boxe 10 by $n^{1}$ and compute that $1^{m} \otimes n^{1}=1^{m} n^{1} \oplus 1^{m-1}(n+1)^{1}$ if $m \geq 1, n \geq 1, n \leq 4$. Thus

$$
\begin{aligned}
& \bigoplus_{j=0}^{4} \ominus^{j} \operatorname{Sym}^{i-j}(V) \otimes \Lambda^{j}(V)=\bigoplus_{j=0}^{4} \ominus^{j} 1^{i-j} \otimes j^{1} \\
& =1^{i} \oplus \bigoplus_{j=1}^{3} \ominus^{j}\left[1^{i-j} j^{1} \oplus 1^{i-(j+1)}(j+1)^{i}\right] \oplus 1^{i-4} \otimes 4^{1}=0
\end{aligned}
$$

if $i \geq 1$. Thereby we have established that our assumption (C.4) implies the result (C.6).

In order to complete our proof of equation (C.6) we still need to verify our assumption (C.4). Let us observe that the bosonic branching functions $\psi^{B}$ possess the same symmetry property, because, since the bosonic characters $\chi^{B}$ are simply products of $\operatorname{sl}(2)$ characters $\chi_{j}=-\chi_{-j-1}$, the identity (C.4) holds trivially for $\psi^{B}$ instead of $\psi^{K}$. We can use this fact to show

$$
\begin{aligned}
\psi_{\omega_{m}(\lambda)}^{K} & =\sum_{i=0}^{\infty}(-1)^{i} \sum_{\beta \in \operatorname{Sym}^{i}\left(g_{-1}\right)} \psi_{\omega_{m}(\lambda)-\beta}^{B}=\sum_{i=0}^{\infty}(-1)^{i} \sum_{\beta \in \operatorname{Sym}^{i}\left(g_{-1}\right)} \psi_{\omega_{m}\left(\lambda-\tilde{\omega}_{m}(\beta)\right)}^{B} \\
& =-\sum_{i=0}^{\infty}(-1)^{i} \sum_{\beta \in \operatorname{Sym}^{i}\left(g_{-1}\right)} \psi_{\lambda-\tilde{\omega}_{m}(\beta)}^{B}=-\sum_{i=0}^{\infty}(-1)^{i} \sum_{\beta \in \operatorname{Sym}^{i}\left(g_{-1}\right)} \psi_{\lambda-\beta}^{B} .
\end{aligned}
$$

The labels $\omega_{2}(\lambda)$ and $\tilde{\omega}_{2}(\lambda)$ were introduced as $\omega_{2}(\lambda)=\left(j_{1},-j_{2}-1, j_{3}\right)$ and $\tilde{\omega}_{2}(\lambda)=$ $\left(j_{1},-j_{2}, j_{3}\right)$ for all $\lambda=\left(j_{1}, j_{2}, j_{3}\right)$. Similar conventions apply to $\omega_{3}$ and $\tilde{\omega}_{3}$.

As we have noted before, the functions $\psi_{\Lambda}^{K}$ can have Laurent expansions with negative coefficients. Such negative coefficients only appear in the atypical sector and they can be traced back to the fact that we expanded the partition function $Z$ in terms of 'unphysical'

\footnotetext{
${ }^{10}$ Since we work with a four-dimensional space $V, 4^{1}=0^{1}$ must denote the trivial one-dimensional space.
} 
characters of Kac modules rather than through those of irreducible representations. The relation between Kac modules and irreducible representation has direct implications on the corresponding branching functions. In fact, the branching functions $\psi_{\lambda}$ that are defined through a decomposition into characters of irreducible representations are related to the branching functions $\psi^{K}$ by $\psi_{\left[j_{1}, j_{2}, j_{3}\right]}(q)=\sum_{\Lambda} \psi_{\Lambda}^{K}(q)$. On the right hand side the summation extends over all those Kac modules $K_{\Lambda}$ that contain the irreducible representation $\left[j_{1}, j_{2}, j_{3}\right]$ in their decomposition series. All relevant decomposition series were spelled out in eq. (A.6). This gives

$$
\begin{aligned}
& \psi_{\lambda_{0,0}}(q)=\psi_{\lambda_{0,0}}^{K}(q)+\psi_{\lambda_{0,2}}^{K}(q) \\
& \psi_{\lambda_{0, l}}(q)=\psi_{\lambda_{0, l}}^{K}(q)+\psi_{\lambda_{0, l+1}}^{K}(q) \quad \forall l \geq 1 \\
& \psi_{\lambda_{k, 0}}(q)=\psi_{\lambda_{k, 0}}^{K}(q)+\psi_{\lambda_{k, 1}}^{K}(q)+\psi_{\lambda_{k,-1}}^{K}(q) \quad \forall k \geq 1 \\
& \psi_{\lambda_{k, l}}(q)=\psi_{\lambda_{k, l}}^{K}(q)+\psi_{\lambda_{k, l+1}}^{K}(q) \quad \forall k \geq 1, l \geq 1 \\
& \psi_{\lambda_{k, l}}(q)=\psi_{\lambda_{k, l}}^{K}(q)+\psi_{\lambda_{k, l-1}}^{K}(q) \quad \forall k \geq 1, l \leq-1 .
\end{aligned}
$$

Let us stress that the branching functions $\psi_{\Lambda}(q)$ for irreducible representations of osp $(4 \mid 2)$ are guaranteed to have non-negative integral coefficients.

\section{A free field construction for $\widehat{\operatorname{osp}}(\mathrm{M} \mid 2 \mathrm{~N})_{1}$}

This appendix contains a free field construction of the affine $\operatorname{osp}(\mathrm{M} \mid 2 \mathrm{~N})$ algebra at level $k=1$ in terms of free fermions and several bosonic ghost systems. Let us decompose all supermatrices $X \in \operatorname{osp}(M \mid 2 N)$ into blocks according to

$$
X=\left(\begin{array}{c|cc}
\mathcal{E} & \overline{\mathcal{T}} & \mathcal{T} \\
\hline-\mathcal{T}^{t} & \mathcal{F} & \mathcal{G} \\
\overline{\mathcal{T}}^{t} & \overline{\mathcal{G}} & -\mathcal{F}^{t}
\end{array}\right)
$$

where $\mathcal{E}$ is antisymmetric and $\mathcal{G}, \overline{\mathcal{G}}$ are symmetric. A basis for the various blocks in the supermatrix $X$ is provided by

$$
\begin{aligned}
& \mathcal{E}_{i j}=e_{i j}-e_{j i} \quad 1 \leq i<j \leq M \\
& \mathcal{F}_{a b}=e_{a b} \quad 1 \leq a, b \leq N \\
& \mathcal{G}_{a b}=\overline{\mathcal{G}}_{a b}=e_{a b}+e_{b a} \quad 1 \leq a \leq b \leq N \\
& \mathcal{T}_{i a}=\overline{\mathcal{T}}_{i a}=e_{i a} \quad 1 \leq i \leq M, 1 \leq a \leq N
\end{aligned}
$$

where $e_{m n}$ are elementary matrices. The matrices we have just introduced describe the various blocks in the supermatrix $X$. We agree to denote by $E_{i j}$ the supermatrix of the form (D.1) where $\mathcal{E}$ is given by $\mathcal{E}_{i j}$ and all other blocks vanish. The basis elements $F_{a b}, G_{a b}, \bar{G}_{a b}, T_{i a}, \bar{T}_{i a}$ are defined similarly. 
Now let us introduce $M$ free fermions $\psi_{i}$ and $2 N$ bosons $\beta_{a}, \gamma_{a}$ with the following basic operator products,

$$
\psi_{i}(z) \psi_{j}(w) \sim \frac{\delta_{i j}}{z-w}, \quad \beta_{a}(z) \gamma_{b}(w) \sim-\gamma_{a}(z) \beta_{b}(w) \sim \frac{\delta_{a b}}{z-w} .
$$

We can define the free field representation of the $\operatorname{osp}(\mathrm{M} \mid 2 \mathrm{~N})$ current algebra through

$$
\begin{aligned}
E_{i j}(z) & =\left(\psi_{i} \psi_{j}\right)(z), & F_{a b}(z) & =-\left(\beta_{a} \gamma_{b}\right)(z) \\
G_{a b}(z) & =\left(\beta_{a} \beta_{b}\right)(z), & \bar{G}_{a b}(z) & =-\left(\gamma_{a} \gamma_{b}\right)(z) \\
T_{i a}(z) & =i\left(\psi_{i} \beta_{a}\right)(z), & & \bar{T}_{i a}(z)=-i\left(\psi_{i} \gamma_{a}\right)(z) .
\end{aligned}
$$

The invariant bilinear form for $\operatorname{osp}(\mathrm{M} \mid 2 \mathrm{~N})$ is $(X, Y)=\frac{1}{2} \operatorname{str}(X Y)$. On the basis elements it takes the following from

$$
\begin{aligned}
\left(E_{i j}, E_{k l}\right) & =-\delta_{i k} \delta_{j l} \quad i<j \text { and } k<l \\
\left(F_{a b}, F_{c d}\right) & =-\delta_{a d} \delta_{b c} \\
\left(G_{a b}, \bar{G}_{c d}\right) & =-\delta_{a c} \delta_{b d} \quad \text { for } a \neq b \text { and } c \neq d \quad\left(G_{a a}, \bar{G}_{b b}\right)=-2 \delta_{a b} \\
\left(T_{i a}, \bar{T}_{j b}\right) & =\delta_{i j} \delta_{a b} .
\end{aligned}
$$

With the help of this form and assuming that $M \neq 2 N+1$, the holomorphic part of the energy momentum tensor is given by the Sugawara construction

$$
\begin{aligned}
T(z)= & \frac{\left(J^{\mu} J_{\mu}\right)(z)}{2\left(k+g^{\vee}\right)}=\frac{1}{2\left(k+g^{\vee}\right)}\left[-\sum_{i<j=1}^{M}\left(E_{i j}^{2}\right)-\sum_{a, b=1}^{N}\left(F_{a b} F_{b a}\right)-\sum_{a<b=1}^{N}\left(\left\{G_{a b}, \bar{G}_{a b}\right\}\right)\right. \\
& \left.-\frac{1}{2} \sum_{a=1}^{N}\left(\left\{G_{a a}, \bar{G}_{a a}\right\}\right)-\sum_{i=1}^{M} \sum_{a=1}^{N}\left(\left[T_{i a}, \bar{T}_{i a}\right]\right)\right] \\
= & -\frac{1}{2} \sum_{i=1}^{M}\left(\psi_{i} \partial \psi_{i}\right)+\frac{1}{2} \sum_{a=1}^{N}\left(\left(\beta_{a} \partial \gamma_{a}\right)-\left(\gamma_{a} \partial \beta_{a}\right)\right)
\end{aligned}
$$

Here, the dual Coxeter number is given by $g^{\vee}=M-2 N-2$ and the value of the level is $k=1$. The central charge of the system is easily seen to take the value $c=\frac{M}{2}-N$.

Let us now introduce the involutive automorphism $\Omega$ such that the fixed point set $\{X \in$ $\operatorname{osp}(M \mid 2 N) \mid \Omega(X)=X\}$ is isomorphic to $\operatorname{osp}(M-1 \mid 2 N)$. On the basis we introduced above, $\Omega$ acts non-trivially only on $E_{i j}, T_{i a}, \bar{T}_{i a}$. In fact, it multiplies all operators with $i=1$ by -1 and leaves the others invariant. If we denote the anti-holomorphic fields corresponding 
to $\psi_{i}, \beta_{a}, \gamma_{a}$ by $\bar{\psi}_{i}, \bar{\beta}_{a}, \bar{\gamma}_{a}$, the deformation operator $J^{\mu} \Omega\left(\bar{J}_{\mu}\right)$ can then be written as

$$
\begin{aligned}
J^{\mu} \Omega\left(\bar{J}_{\mu}\right)= & -\sum_{i<j=1}^{M} \varpi_{i}\left(\psi_{i} \psi_{j}\right)\left(\bar{\psi}_{i} \bar{\psi}_{j}\right)-\sum_{a, b=1}^{N}\left(\beta_{a} \gamma_{b}\right)\left(\bar{\beta}_{b} \bar{\gamma}_{a}\right) \\
& +\sum_{a<b=1}^{N}\left[\left(\beta_{a} \beta_{b}\right)\left(\bar{\gamma}_{a} \bar{\gamma}_{b}\right)+\left(\gamma_{a} \gamma_{b}\right)\left(\bar{\beta}_{a} \bar{\beta}_{b}\right)\right]+\frac{1}{2} \sum_{a=1}^{N}\left[\left(\beta_{a} \beta_{a}\right)\left(\bar{\gamma}_{a} \bar{\gamma}_{a}\right)+\left(\gamma_{a} \gamma_{a}\right)\left(\bar{\beta}_{a} \bar{\beta}_{a}\right)\right] \\
& -\sum_{i=1}^{M} \sum_{a=1}^{N} \varpi_{i}\left[\left(\psi_{i} \beta_{a}\right)\left(\bar{\psi}_{i} \bar{\gamma}_{a}\right)-\left(\psi_{i} \gamma_{a}\right)\left(\bar{\psi}_{i} \bar{\beta}_{a}\right)\right] \\
= & \frac{1}{2}\left[\sum_{i=1}^{M} \varpi_{i} \psi_{i} \bar{\psi}_{i}+\sum_{a=1}^{N}\left(\gamma_{a} \bar{\beta}_{a}-\beta_{a} \bar{\gamma}_{a}\right)\right]^{2}
\end{aligned}
$$

where $\varpi=(-1,1, \ldots, 1)$. In order for the last line of (D.6) to make sense, we need to first expand the square and then bring all the fields in the standard normal ordering.

\section{References}

[1] K. Pohlmeyer, Integrable Hamiltonian Systems and Interactions Through Quadratic Constraints, Commun. Math. Phys. 46 (1976) 207-221.

[2] M. Lüscher and K. Pohlmeyer, Scattering of massless lumps and nonlocal charges in the two-dimensional classical nonlinear sigma model, Nucl. Phys. B137 (1978) 46.

[3] M. Lüscher, Quantum nonlocal charges and absence of particle production in the two-dimensional nonlinear sigma model, Nucl. Phys. B135 (1978) 1-19.

[4] E. Brezin, C. Itzykson, J. Zinn-Justin and J. B. Zuber, Remarks about the existence of nonlocal charges in two-dimensional models, Phys. Lett. B82 (1979) 442-444.

[5] H. Eichenherr and M. Forger, On the dual symmetry of the nonlinear sigma models, Nucl. Phys. B155 (1979) 381.

[6] H. Eichenherr and M. Forger, Higher local conservation laws for nonlinear sigma models on symmetric spaces, Commun. Math. Phys. 82 (1981) 227.

[7] J. H. Schwarz, Classical symmetries of some two-dimensional models, Nucl. Phys. B447 (1995) 137-182 hep-th/9503078.

[8] J. M. Evans and A. J. Mountain, Commuting charges and symmetric spaces, Phys. Lett. B483 (2000) 290-298 hep-th/0003264.

[9] Y. Y. Goldschmidt and E. Witten, Conservation laws in some two-dimensional models, Phys. Lett. B91 (1980) 392. 
[10] E. Abdalla, M. Forger and M. Gomes, On the origin of anomalies in the quantum nonlocal charge for the generalized nonlinear sigma models, Nucl. Phys. B210 (1982) 181.

[11] R. R. Metsaev and A. A. Tseytlin, Type IIB superstring action in $A d S_{5} \times S^{5}$ background, Nucl. Phys. B533 (1998) 109-126 hep-th/9805028.

[12] N. Berkovits, Super-Poincaré covariant quantization of the superstring, JHEP 04 (2000) 018 hep-th/0001035.

[13] G. Arutyunov and S. Frolov, Superstrings on $A d S_{4} \times C P^{3}$ as a coset sigma-model, 0806.4940 .

[14] P. Fre and P. A. Grassi, Pure Spinor Formalism for Osp $(N \mid 4)$ backgrounds, 0807.0044.

[15] N. Berkovits, C. Vafa and E. Witten, Conformal field theory of AdS background with Ramond-Ramond flux, JHEP 03 (1999) 018 hep-th/9902098.

[16] M. Bershadsky, S. Zhukov and A. Vaintrob, PSL $(n \mid n)$ sigma model as a conformal field theory, Nucl. Phys. B559 (1999) 205-234 hep-th/9902180.

[17] M. R. Zirnbauer, Conformal field theory of the integer quantum Hall plateau transition, hep-th/9905054

[18] N. Read and H. Saleur, Exact spectra of conformal supersymmetric nonlinear sigma models in two dimensions, Nucl. Phys. B613 (2001) 409 hep-th/0106124.

[19] A. Babichenko, Conformal invariance and quantum integrability of sigma models on symmetric superspaces, Phys. Lett. B648 (2007) 254-261 hep-th/0611214.

[20] N. Mann and J. Polchinski, Finite density states in integrable conformal field theories, in From Fields to Strings: Circumnavigating Theoretical Physics, pp. 1365-1383. 2004. hep-th/0408162.

[21] N. Mann and J. Polchinski, Bethe ansatz for a quantum supercoset sigma model, Phys. Rev. D72 (2005) 086002 hep-th/0508232.

[22] T. Quella, V. Schomerus and T. Creutzig, Boundary spectra in superspace sigma-models, arXiv:0712.3549 [hep-th].

[23] C. Candu and H. Saleur, A lattice approach to the conformal OSp $(2 S+2 \mid 2 S)$ supercoset sigma model. Part I: Algebraic structures in the spin chain. The Brauer algebra, arXiv:0801.0430 [hep-th].

[24] C. Candu and H. Saleur, A lattice approach to the conformal OSp $(2 S+2 \mid 2 S)$ supercoset sigma model. Part II: The boundary spectrum, arXiv:0801.0444 [hep-th]. 
[25] E. Witten, Nonabelian bosonization in two dimensions, Commun. Math. Phys. 92 (1984) 455-472.

[26] A. N. Schellekens and S. Yankielowicz, Simple currents, modular invariants and fixed points, Int. J. Mod. Phys. A5 (1990) 2903-2952.

[27] P. H. Ginsparg, Applied conformal field theory, in Fields, Strings and Critical Phenomena (E. Brézin and J. Z. Justin, eds.), Les Houches, Session XLIX, 1988. hep-th/9108028

[28] F. Lesage, P. Mathieu, J. Rasmussen and H. Saleur, The $\widehat{s u}(2)_{-\frac{1}{2}} W Z W$ model and the

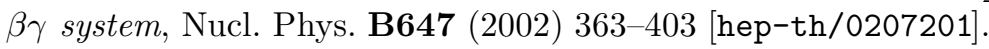

[29] V. Schomerus and H. Saleur, The GL(1|1) WZW model: From supergeometry to logarithmic CFT, Nucl. Phys. B734 (2006) 221-245 hep-th/0510032.

[30] H. Saleur and V. Schomerus, On the $S U(2 \mid 1)$ WZW model and its statistical mechanics applications, Nucl. Phys. B775 (2007) 312-340 hep-th/0611147.

[31] G. Götz, T. Quella and V. Schomerus, The WZNW model on PSU (1, 1|2), JHEP 03 (2007) 003 hep-th/0610070.

[32] T. Quella and V. Schomerus, Free fermion resolution of supergroup WZNW models, arXiv:0706.0744 [hep-th].

[33] F. Lesage, P. Mathieu, J. Rasmussen and H. Saleur, Logarithmic lift of the $\widehat{s u}(2)_{-1 / 2}$ model, Nucl. Phys. B686 (2004) 313 arXiv:hep-th/0311039.

[34] J. L. Cardy, Boundary conditions, fusion rules and the Verlinde formula, Nucl. Phys. B324 (1989) 581.

[35] P. Di Francesco, P. Mathieu and D. Senechal, Conformal Field Theory. Graduate Texts in Contemporary Physics. Springer, New York, 1999.

[36] L. Frappat, P. Sorba and A. Sciarrino, Dictionary on Lie algebras and superalgebras. Academic Press Inc., San Diego, CA, 2000. Extended and corrected version of the E-print hep-th/9607161.

[37] R. Gopakumar, From free fields to AdS, Phys. Rev. D70 (2004) 025009 hep-th/0308184.

[38] O. Aharony, J. R. David, R. Gopakumar, Z. Komargodski and S. S. Razamat, Comments on worldsheet theories dual to free large $N$ gauge theories, Phys. Rev. D75 (2007) 106006 hep-th/0703141.

[39] N. Berkovits, Perturbative Super-Yang-Mills from the topological $A d S_{5} \times S^{5}$ sigma model, 0806.1960 . 
[40] J. Germoni, Indecomposable representations of osp $(3 \mid 2), D(2,1 ; \alpha)$ and $G(3)$, Bol. Acad. Nac. Cienc. (Córdoba) 65 (2000) 147-163. Colloquium on Homology and Representation Theory (Spanish) (Vaquerías, 1998).

[41] J. Van Der Jeugt, Irreducible representations of the exceptional Lie superalgebras $D(2,1 ; \alpha)$, J. Math. Phys. 26 (1985) 913-924. 